\title{
Analysis of Reversible Switching Capacitive DAC Based Low Power SAR-ADC
}

Prathiba G ( $\square$ prathibag424@gmail.com )

University College of Engineering

Shanthi M

Saranathan College of Engineering

Manuscript

Keywords: Parasitic Effect, Reversible Switching, Conversion Linearity, Accuracy

Posted Date: February 5th, 2021

DOI: https://doi.org/10.21203/rs.3.rs-164633/v1

License: (c) (i) This work is licensed under a Creative Commons Attribution 4.0 International License. Read Full License 


\title{
Analysis of Reversible Switching Capacitive DAC Based Low Power SAR-ADC
}

\author{
G.Prathiba ${ }^{\mathrm{a}}$, M.Santhi ${ }^{\mathrm{b}}$ \\ ${ }^{a}$ G.Prathiba, Department of ECE, University College of Engineering, Ariyalur, Tamil Nadu, \\ India \\ *Corresponding mail: prathibag424@gmail.com \\ ${ }^{b}$ M.Santhi, Department of ECE, Saranathan College of Engineering, Trichy, Tamil Nadu, \\ India
}

mail: santhimphd@gmail.com

\begin{abstract}
This paper presents an analysis of Reversible Switching Capacitive Digital to Analog converter (RSC-DAC) based low power Successive Approximation Register Analog to Digital Converter (SAR-ADC).The proposed structure involves, the QVDC (Quantum Voltage Differential Comparator) constructed using Simple Transconductance Amplifier (STA) technique , the RSC-DAC switching energy reduced by 93\% contrast to the standard Charge Redistribution Switching Capacitive DAC (CRSC-DAC) method, and the Successive Approximation Register(SAR) control logic is designed with D-FF based shift register. The QVDC comparator allows very small voltage comparison, and consumes low power and area effective. The linearity parameters such as Integral Nonlinearity, Differential Nonlinearity and parasitic effect of the capacitor of the RSC-DAC is analyzed and improved by the new approach is named as Adaptive Random Code Generation (ARCG) Technique. The above overall design is implemented by TANNER-EDA tool in 250nm CMOS technology, consumes $1.74 \mathrm{~mW}$ power at $60 \mathrm{MS} / \mathrm{s}$. The INL and DNL of the proposed structure is $+0.18 /-$ 0.12 LSB and +0.11/-0.05 LSB respectively.
\end{abstract}

Keywords: Parasitic Effect, Reversible Switching, Conversion Linearity, Accuracy. 


\section{Introduction}

Successive Approximation Register - Analog to Digital Converter (SAR-ADC), as an alternative method to the pipelined ADC has become famous for battery-powered versatile applications, such as a serial data communication link, mobile communication and Remote sensor network which need high speed and standard-resolution (8-16 bit). SAR- ADCs achieve very low power consumption due to their simple architecture and operation. The existing SAR-ADC utilizes binary-weighted CRSC-DAC structure. Nonetheless, the SAR switching relies basically upon the performance of a capacitive DAC that deducts the reference voltage from the information signal. Capacitor mismatches, and parasitic of the capacitive-DAC influence the transformation accuracy. In any case, the capacitance of the DAC exhibit increments dramatically based on the resolution, which forces larger utilization of switching energy, area, and settling time. The switching arrangements of the DAC array where the traditional charge redistribution switching results bring about more terrible change linearity and more energy misfortunes.

An RSC-DAC (Reversible Switching Capacitive-Digital to Analog Converter) based SAR-ADC has been as of late proposed, which accomplishes a huge switching energy saving when contrasted and the customary (CRSC-DAC) approach. This paper inspects the conversion nonlinearities, incited by supply noise, switching strategies, and parasitic impacts in SAR ADCs. The static nonlinearities dependent on the switching method are analyzed, and check the adequacy of the Reversible Switching Capacitive-DAC approach. The newly constructed RSC-DAC switching shows the presentation benefits regarding power utilization and linearity. 


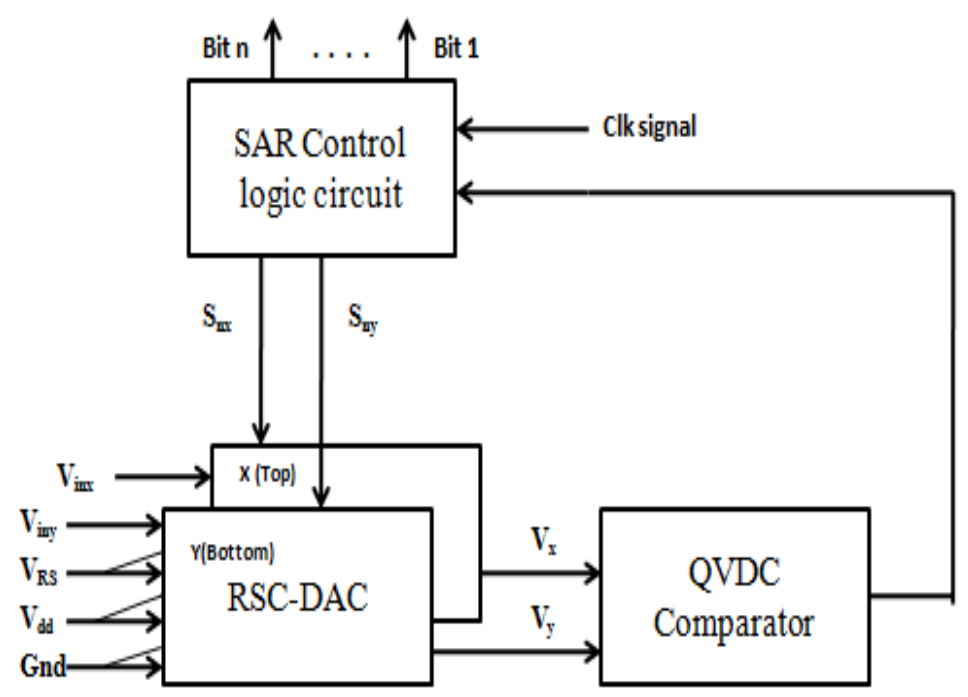

Figure 1: Proposed SAR-ADC Architecture

The resting of this proposal is formulated as follows. Part 2 explores about related work. Division 3 investigates SAR-ADC architecture with proposed RSC-DAC, QVDC comparator and SAR control logic. Segment 4 explains about Non-binary Conversion algorithm and ARCG (Adaptive Random Code Generator) of the proposed work. The analysis of results and conclusion are detailed in Portion 5 and 6 respectively.

\section{Related Work}

A predictable successive approximation register (SAR) ADCs [1], the primary sources of power debauchery are the Sample and hold circuit, comparator and the DAC capacitor selection. The digital power reduces with the development of knowledge. Notwithstanding, the control of the comparator and capacitor network is defective by in greatness and noise issues. The ADC [2] adopts a powerful, well-organized amplifier distribution architecture in which supplementary switches are acquainted with lessening the crosstalk between the two operation amplifier allocation successive stages. Another example is used in the main phase of the ADC to keep away from utilizing an excited Sample-and-Hold Amplifier circuit at the 
information and to avoid the relating imperative between the primary Multiplying Digital-toAnalog Converter and flash input sign paths.

The architectural idea of a most positive sub ranging ADC got with the tumble of a Flash and a SAR, which is additionally investigated during its sensible design and test verification [3]. The answer doubles the best possible speed of activity of the SA- ADCs at the qualified low force cost of a low-resolution Flash. The digital modification method and a capacitorbased DAC make certain challenging necessities for the Flash. The charge compensation technique [4] with voltage feedback for split DAC design in SAR ADCs. The charge pays is accomplished by utilizing an open loop amplifier that performs voltage input to the DAC display through a repayment capacitor, which is simple to implement with extremely low force dissemination.

An energy- productive charge reusing method adopted for a SAR converter [5] with capacitive DAC. The exploits of the supply voltage as the suggestion of the ADC would guide to a signal, hang of the converter that is wider than the characteristic signal range; this would shrink the efficient SNR. This drawback is detached with the proposed technique that grants a submissive gain of 2 of the inputs. A reiteration of the LSB that loosens up the limitations of noise and make up for additionally brings down the force utilization of the comparator. The idea of an ideal sub ranging ADC [6] acquired with the course of a Flash and a SAR, which likewise provided the ideal speed of activity of the SAR ADCs at the generally low force and low cost. The digital correction strategy and a capacitor-based DAC guarantee not to demanding requirements for the Flash Measured DNL and INL are $+0.87 /-0.81 \mathrm{LSB}$ and $+0.71 /-1.55 \mathrm{LSB}$, respectively. The comparator power relies upon the resolution. For a several $\mathrm{mV}$, simply a latch makes the perfect comparator. 
A charge compensation methodology with voltage feedback design [7] is introduced to forestall the conversion nonlinearity because of the parasitic impact of split capacitive DAC structure in successive approximation ADCs. The method is used in the plan of an $80 \mathrm{MS} / \mathrm{s}$ SAR ADC with $10 \mathrm{~b}$ in the $65-\mathrm{nm}$ CMOS circuit. Another strategy in the DAC capacitor array of SA ADC for switching the capacitors introduced [8] by separating the MSB capacitor into $b-1$ binary scaled sub-capacitors, and the normal switching energy of the array can be decreased by $37 \%$ contrasted with the conventional switching strategy. A Novel self-timing technique based switch-driving registers proposed for a rapid Successive Approximation Register. This circuit can give quick charging way from comparator yield to DAC array and accumulate the comparison outcome at the same time at each guess Bit-cycle. The processing delay of the overall work is about 60ps just in a 90nm CMOS process. By utilizing this procedure, the $5 \mathrm{~b}$ SAR ADC accomplishes $30.3 \mathrm{~dB}$ SNDR with power consumption is $10.5 \mathrm{~mW}$ at $285 \mathrm{MS} / \mathrm{s}$ high sampling-rate [9].

\section{Proposed SAR-ADC Architecture}

\subsection{Overview}

Figure. 1 shows the proposed 8 bit SAR- ADC architecture. The proposed SAR-ADC consists of fewer blocks such as one QVDC comparator, one SAR control logic circuit and the RSC-DAC array. In this design a very special counter logic circuit is used known as a successive-approximation register (SAR) control logic circuit. Rather than including up in binary sequence, the register counts of attempting all estimations of bits beginning with the MSB (most-significant bit) and completing at the LSB (least-significant bit). The way in which the register logic circuit counts are indistinguishable from the "trial-and-fit" strategy for decimal-to-binary transformation. The SAR logic design mainly utilizes the BSA (Binary 
Search Algorithm). The essential algorithm of binary search is to convert the analog signal into digital signal/quantized structure. The conversion process starts after discharging the capacitors in RSC-DAC. During sampling mode capacitor array is charged by $\mathrm{V}_{\text {in }}$ (input source voltage). During charge redistribution mode, MSB (Most Significant Bit) set to one and remaining bits set to zero.The SAR control logic incorporates shift registers and logic switch drivers which restraint the DAC activity by playing out a BSA during the transformation cycle. A Reference Buffer Free (RBF) technique is utilized to reduce the power dissemination and improve the DAC settling period. As the supplies $V_{\mathrm{dd}}$ and Gnd are utilized straightforwardly as reference voltages, the transformation sensitivity to supply variation is quantitatively dissected.

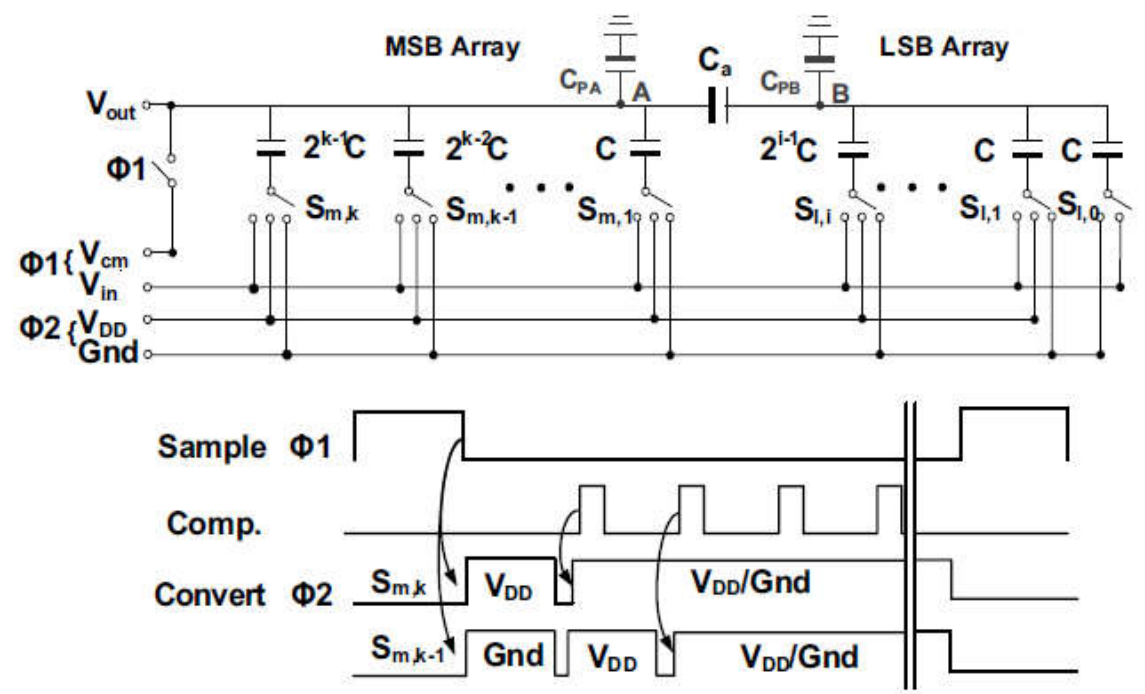

Figure 2: Conventional Charge-Redistribution switching Capacitive DAC

\subsection{Conventional Switching Method}


Figure 2 displays a conventional $n$-bit single-ended CRSC- DAC array structure with $k$-bit most significant bit and $i$-bit least Significant bit sub-array and its switching timing chart. During the sampling time, the information signal $V_{\text {in }}$ is put away in the whole capacitive array structure. Based on the algorithmic conversion at that point starts by switching just the MSB capacitor connected to $V_{\mathrm{dd}}$ and the others connected to Gnd (ground). The switching method of the most-significant capacitor depends on the decision received from the comparator. If comparator output is low the $\mathrm{S}_{\mathrm{m}, \mathrm{k}}$ switch is connected back to Gnd. If comparator output is high, then the $S_{\mathrm{m}, \mathrm{k}}$ switch is kept to $V_{\mathrm{dd}}$. For one or the other choice, all the while, the switch $S_{\mathrm{m}, \mathrm{k}-1}$ (MSB/2) couple to $V_{\mathrm{dd}}$ for the following binary digit comparison. The above cycle will be repeated for the count of $n-1$ cycle. The CRSC-DAC strategy is not extremely power effective, particularly while discharging the MSB and charging the MSB/2 capacitor (bit choice back from "1" to "0"). The capacitance of the DAC circuit increments dramatically depends on the resolution, which forces extensive utilization of switching energy, area, and settling time. The switching arrangements of the DAC circuit where the conventional CRSC-DAC results in poor transformation linearity and more wastage of power [10]. 


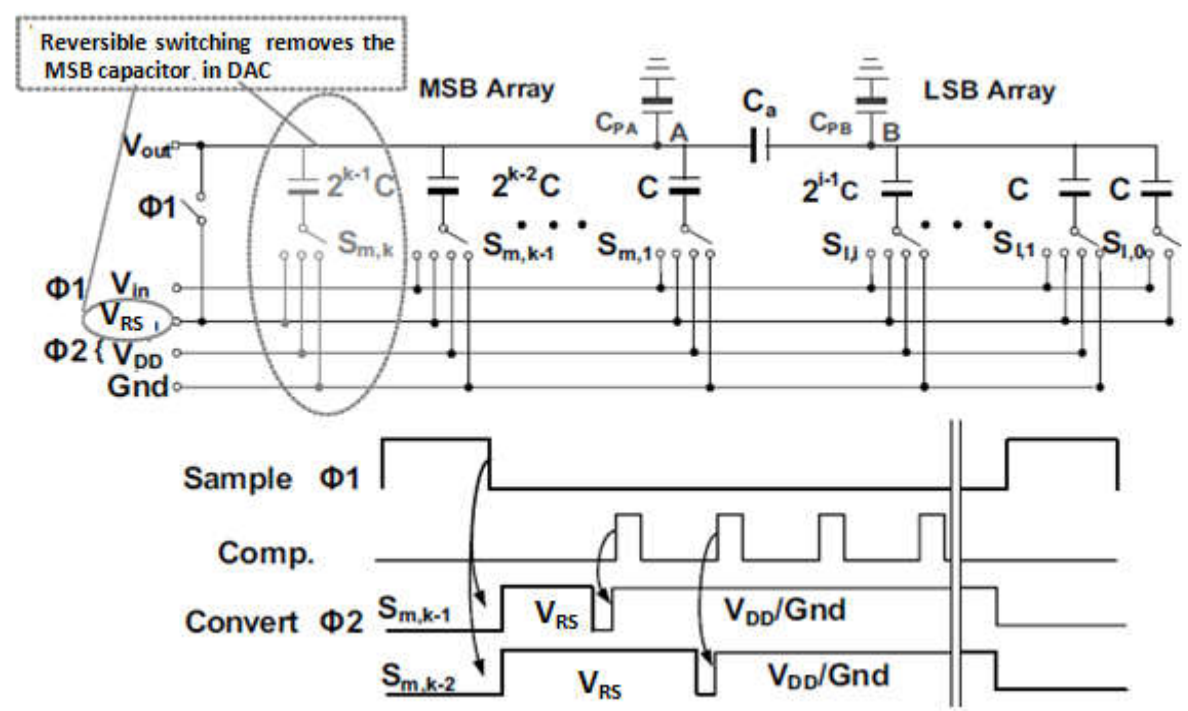

Figure 3: Proposed RSC-DAC

\subsection{Proposed Reversible Switching Capacitive (RSC) -DAC}

The Reversible switching strategy proposed with the array capacitance prompting around 93\% energy, reduced when contrasted and the conventional one. Figure. 3 display the reversible switching capacitive DAC array structure. In the sampling stage $\phi_{1}, V_{\text {in }}$ is stocked in the capacitor of the DAC array. In the course of the transformation phase $\phi_{2}$, all the capacitors bottom-plates are switched to the $V_{\mathrm{RS}}$ first, to offer rise to the voltage $-V_{\text {in }}$ at the yield. The sign indication of $V_{\text {out }}$ decides the MSB as the logic appropriately controls $S_{\mathrm{m}, \mathrm{k}-1}$. On this occasion that $-V_{\text {in }}<0, S_{m, \mathrm{k}-1}$ switch goes to Gnd while different switches $S_{\mathrm{m}, \mathrm{k}-2}, \ldots$, $S_{1,0}$ stay associated with $V_{\mathrm{RS}}$. On the off chance that $-V_{\mathrm{in}}>0, S_{\mathrm{m}, \mathrm{k}-1}$ is changed to $V_{\mathrm{dd}}$. The cycle will be repeated for $n-2$ times. The reversible switching approach performs the MSB transition by connecting the differential arrays to $V_{\mathrm{RS}}$. The power utilization is simply gotten based on what is expected to drive the base plate parasitic of the capacitive DAC arrays, while in the regular charge-redistribution where the fundamental MSB progress from low to high (up) costs prominent switching power and settling period [11]. Besides, as the MSB capacitor is not needed any longer, it very well may be eliminated from the $k$-Bit DAC array. 
Thusly, the accompanying $(k-1)$ Bit estimation is finished with an $(k-1)$ Bit array rather than its $k$-Bit counterpart, prompting a half capacitance decrease regarding the conventional method. Utilizing supplies as reference voltages keep static force dissemination from reference buffers, despite the fact that the change turns out to be sensitive to the supply ripple because of the switching impact [12]. As the reversible switching method, charges $50 \%$ less capacitance, all the while, when contrasted and the conventional switching, it can adequately diminish the under-shoot of the source or reference buffer. An adaptive random code methodology is proposed here to rectify the above constraints and also improve the DNL (Differential Nonlinearity) and INL (Integral Nonlinearity). For the $k$-Bit conventional CRSC- DAC based SAR-ADC; the switching energy is stated in equation (1).

$$
E_{m, k-b i t}=\sum_{i=1}^{k}\left(2^{k+1-2 i}\right) C V_{r}^{2}
$$

The standard switching energy for the $k$-Bit RSC-DAC based SAR-ADC is described in equation (2)

$$
E_{m, k-b i t}=\sum_{i=1}^{k-1}\left(2^{k-2-2 i}\right) C V_{r}^{2}
$$

Where $E_{m, k-b i t}$ is the standard switching energy of the $k$-Bit capacitive DAC

$C$ is the input capacitance of DAC structure

$V_{r}$ is the reference voltage 


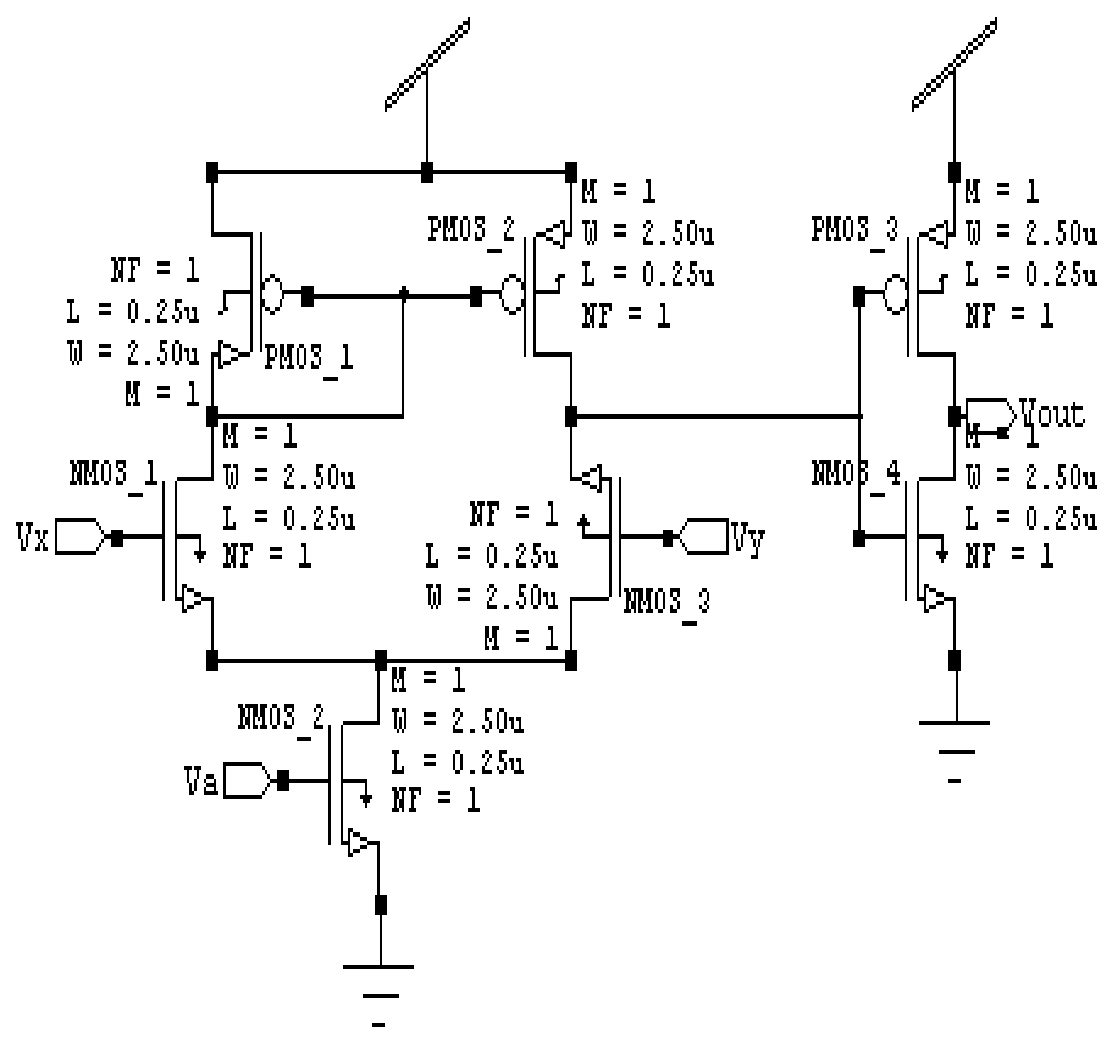

Figure 4: Schematic diagram for QVDC comparator

\subsection{Effect of Parasitic Capacitors on RSC-DAC}

Conventional split capacitor DAC is sensitive to parasitic capacitance at nodes $\mathrm{A}$ and $\mathrm{B}$ [shown in Figure. 3] which causes a mismatch between the array of MSB and the array of the LSB. In the proposed architecture, to limit the unfavourable impacts of parasitic capacitances, their value is taken into account. More specifically, the array of MSB and the LSB, each has a unit capacitor C connected to ground. Assume $\mathrm{C}_{\mathrm{PA}}$ and $\mathrm{C}_{\mathrm{PB}}$ are parasitic capacitance at $\mathrm{A}$ and $\mathrm{B}$, those two unit capacitors can be adjusted to be $\mathrm{C}-\mathrm{C}_{\mathrm{PA}}$ and $\mathrm{C}-\mathrm{C}_{\mathrm{PB}}$. Then the parasitic capacitor and the adjusted capacitor together act as the unit capacitor. MSB array and LSB array are ideally matched if mismatch of individual capacitor is not considered. 


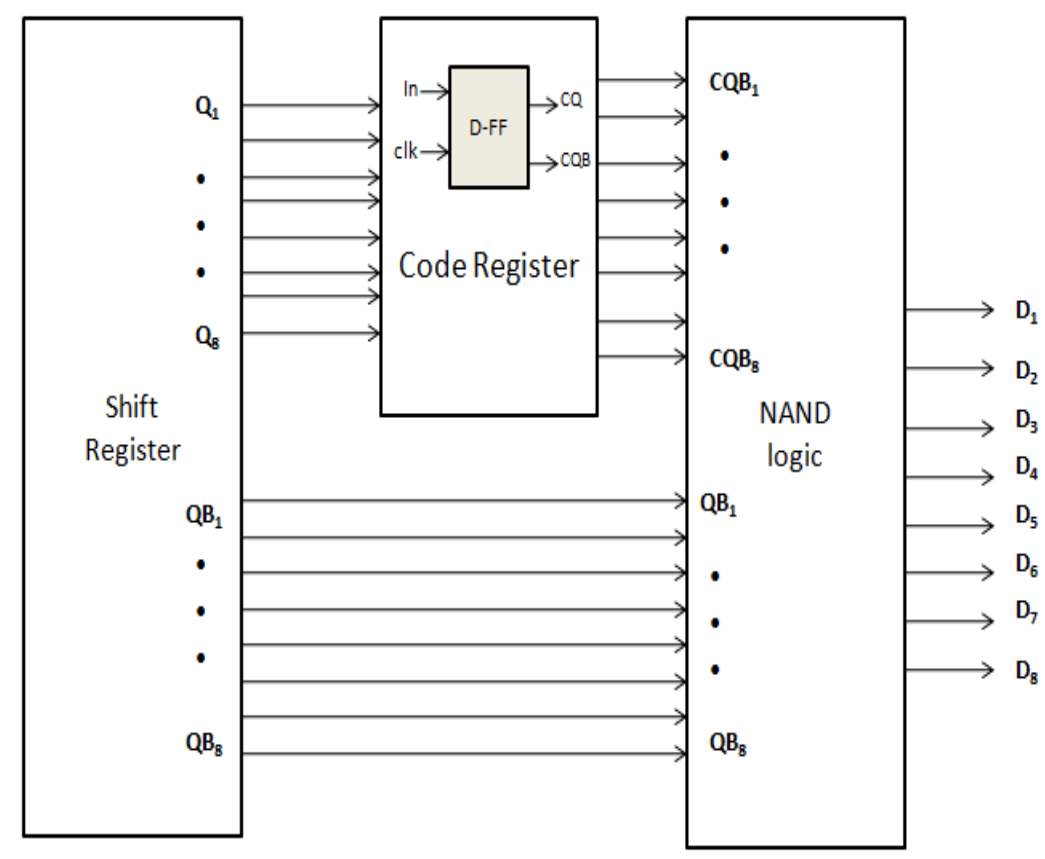

Figure 5: Blocks of SAR control logic

\subsection{Quantum Voltage Differential Comparator (QVDC)}

In the previous TS-OLC (Two Stage-Open Loop Comparator) technique a common source amplifier is used to get the overall gain of the amplifier and the region capped by the common source transistor is increased with the objective of lessening the huge parasitic capacitance, which is the origin of the delay in the first stage. This causes increases in the processing delay and power consumption of the circuit. To rectify the above problems a Quantum Voltage Differential Comparator (QVDC) comparator has been devised from the Simple Transconductance Amplifier (STA) is shown in Figure.4. QVDC is a type of differential voltage comparator. Since this comparator has two inputs for analog signals $V x$ and $V y$, the common mode noise rejection is much better. This is the reason why most ADCs use differential 
comparators. A Simple Transconductance Amplifier (STA) circuit consists of two circuits, a current mirror and a differential pair. By combining the current mirrors at the top and the differential pair at the bottom, STA circuit can be designed. This is commonly used as a transconductance amplifier that generates a current output depending on the difference between the two input voltages. This circuit can also be used as a voltage amplification circuit by taking a voltage at the output instead of a current. The proposed SAR-ADC use this voltage amplifier as a comparator to compare a reference voltage with an input voltage $\left(\mathrm{V}_{\mathrm{x}}\right.$ and $\left.\mathrm{V}_{\mathrm{y}}\right)$, and $\mathrm{V}_{\mathrm{a}}$ is constant voltage. The simple voltage amplifier has been used in the proposed QVDC comparator. In the QVDC comparator schematic circuit only the NMOS1 and NMOS4 are varied, while transistor PMOS1 and PMOS2 are identical. The suggested work based on differential amplifier and the mathematical equation used to obtain the output voltage is represented in (3)

$$
V_{\text {out }}=A_{d}\left(V_{x}-V_{y}\right)
$$

Where $A_{d}$ is the differential mode gain

$V_{x}$ and $V_{y}$ are the input voltages of QVDC (Volts) 


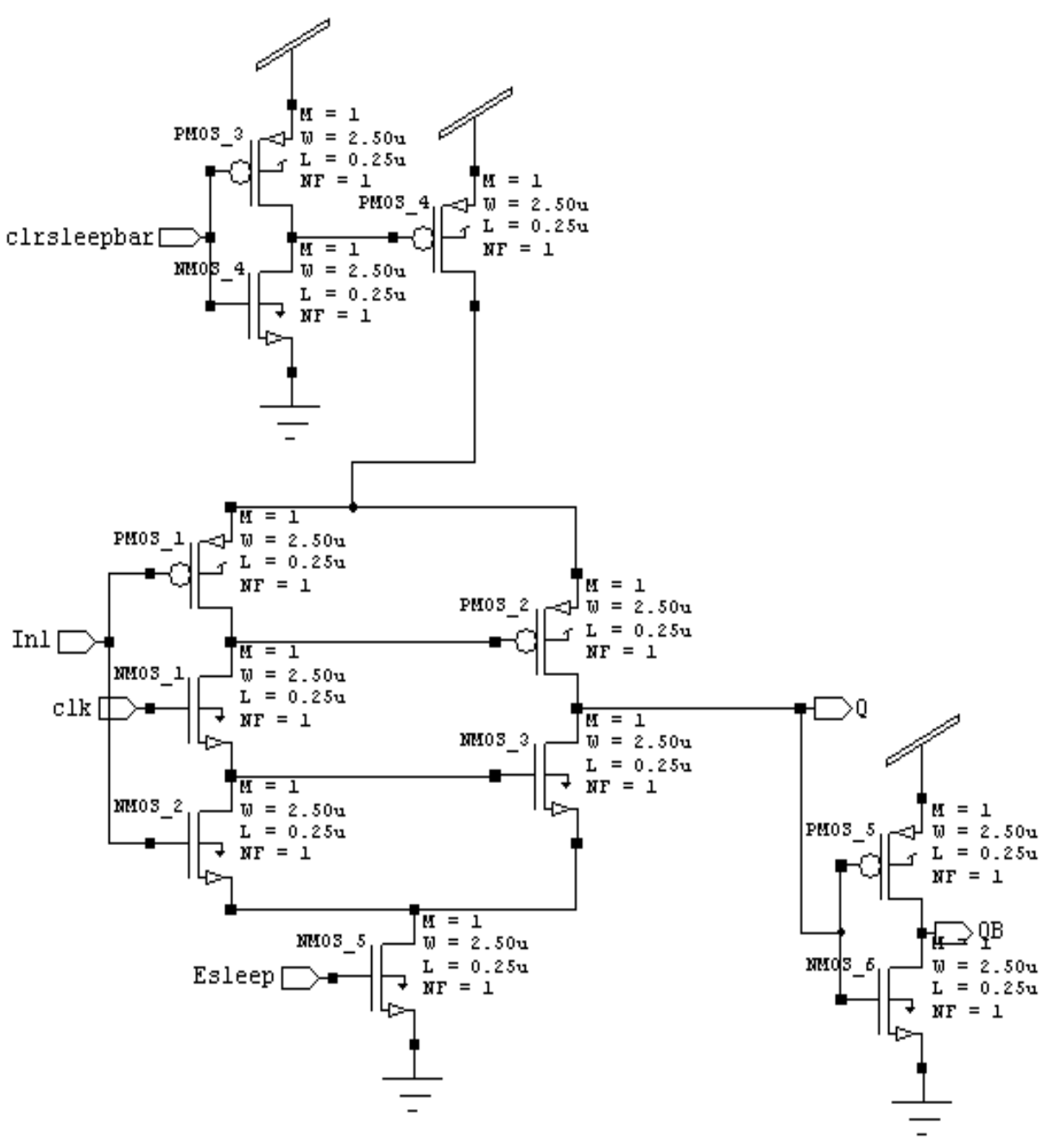

Figure 6: Schematic of D-FF

Table 2: Truth table of D-FF

\begin{tabular}{|c|c|c|c|c|c|c|c|c|c|c|c|}
\hline clk & In1 & ES & P1 & P2 & P4 & N1 & N2 & N3 & N5 & Q & QB \\
\hline High & 0 & 0 & ON & OFF & ON & ON & OFF & ON & OFF & 0 & 1 \\
\hline High & 0 & 1 & ON & OFF & OFF & ON & OFF & OFF & ON & 0 & 1 \\
\hline High & 1 & 0 & OFF & ON & ON & ON & ON & OFF & OFF & 1 & 0 \\
\hline High & 1 & 1 & OFF & ON & OFF & ON & ON & OFF & ON & 1 & 0 \\
\hline Low & 0 & 0 & ON & OFF & ON & OFF & OFF & OFF & OFF & 0 & 1 \\
\hline Low & 0 & 1 & ON & OFF & OFF & OFF & OFF & OFF & ON & 0 & 1 \\
\hline
\end{tabular}




\begin{tabular}{|c|c|c|c|c|c|c|c|c|c|c|c|}
\hline Low & 1 & 0 & OFF & OFF & ON & OFF & ON & OFF & OFF & 0 & 1 \\
\hline Low & 1 & 1 & OFF & ON & OFF & OFF & ON & OFF & ON & 0 & 1 \\
\hline
\end{tabular}

\subsection{Successive Approximation Register (SAR) Control Logic}

Figure.5 shows the blocks on the SAR control logic design uses a Shift Register and code register constructed using D-type flip-flops. From this diagram the $\mathrm{Q}_{1}$ to $\mathrm{Q}_{8}$ outputs of the shift register is fed into the enable process of the code register, while the complementary output $\mathrm{QB}_{1}$ to $\mathrm{QB}_{2}$ of the shift register is fed as one of the inputs of the NAND gates. The complementary output CQB1 to CQB8 of the code register is fed as the other input to the NAND gates. The D-FF configuration used in the code register works in two modes is a high threshold mode and low threshold mode dependent on Esleep (ES). The high threshold mode decreases the power leakage and Low Threshold mode upgrade the speed of the system. The schematic diagram of D-FF is depicted in Figure.6. In the occasion that the clk is high In1 set to 1,ES set to 0 then the transistors, $\mathrm{P} 2, \mathrm{P} 4, \mathrm{~N} 1, \mathrm{~N} 2$, are $\mathrm{ON}$ and $\mathrm{P} 1, \mathrm{~N} 3, \mathrm{~N} 5$ are $\mathrm{OFF}$ subsequently the yields $\mathrm{Q}$ is 1 and $\mathrm{QB}$ is 0 .In the event that the clk is low $\operatorname{In} 1$ set to 0,ES set to 1, then the transistors $\mathrm{P} 1$ and N5are ON and P2,P4,N1,N2,N3 are OFF subsequently the yield Q is 0 and QB is 1, which is delineated in Table 2. P5 and N6 transistors utilized for complement function to give QB. Figure.7 shows the Schematic diagram of Shift register. The output of the QVDC comparator is taken care of to the contribution of the SAR Control Logic which either set as high or reset as low to the output bits in understanding with the binary search algorithm. The QVDC comparator output is contrasted and the bit at the clock when the specific code register is empowered and the output response of the NAND gates gives the output $\mathrm{D}_{1} \mathrm{toD}_{8}$ 


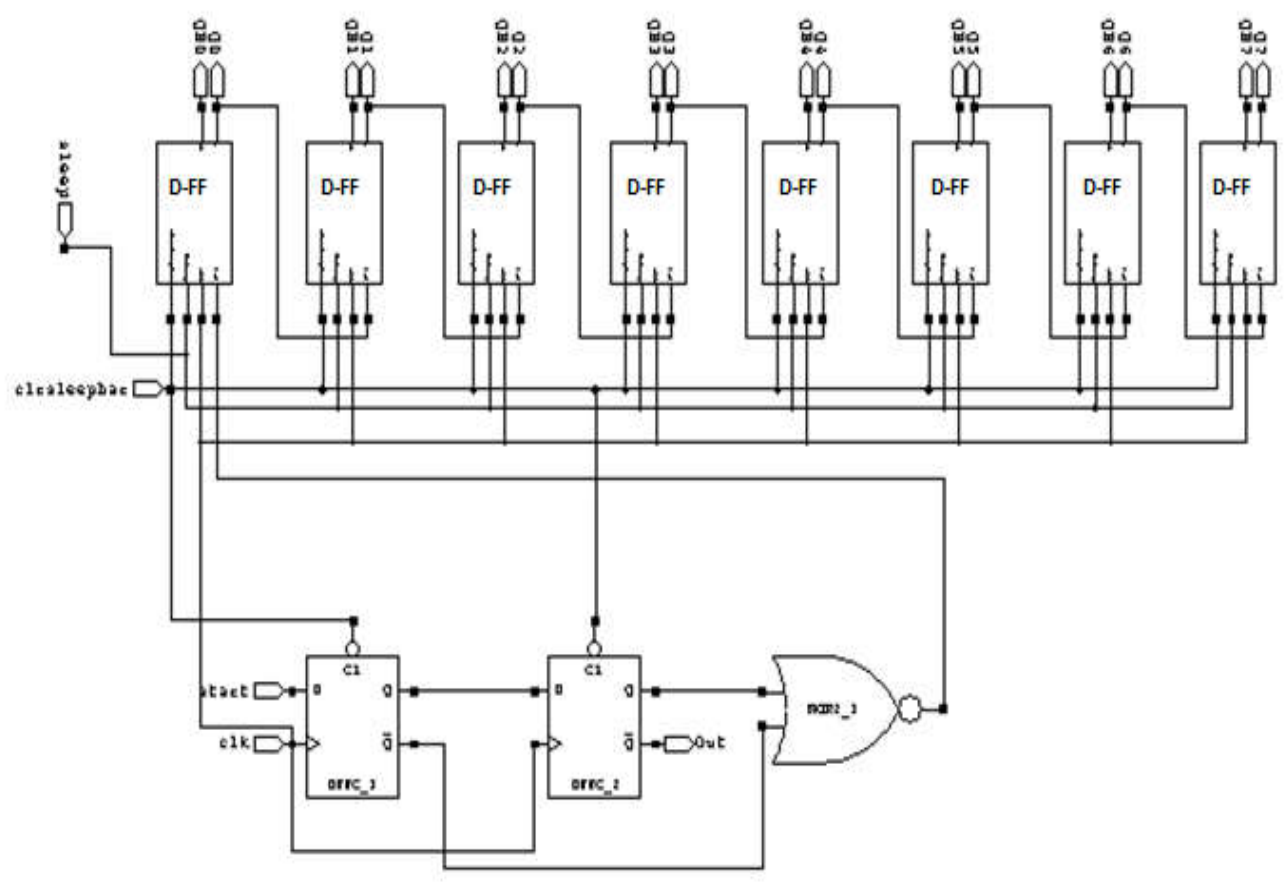

Figure 7: Schematic diagram of Shift register

The SAR-ADC has compacted configuration contrast with flash ADC, which makes highly economical. The actual restriction of SAR ADC is it has only one comparator during the total conversation measure. In the event that there is any make up for the error in the comparator, it will return on the all adaptation bits. However, the static construction errors do not influence dynamic activities of SAR-ADC.

\section{Algorithm}

\subsection{Non Binary Conversion Algorithm}

A successive-approximation ADC utilizes a non binary conversion algorithm to progressively limit a range that contains the input voltage. At each consecutive step, the converter verifies the input voltage to the yield of an internally generated voltage of digital to analog converter which may speak to the centre point of a sortout voltage range [13]. At each progression in this cycle, the approximation is put away in a SAR. The structural outline of 16-Bit non binary conversion algorithm appears in Figure. 8 and the algorithm explained in step by step process below. 


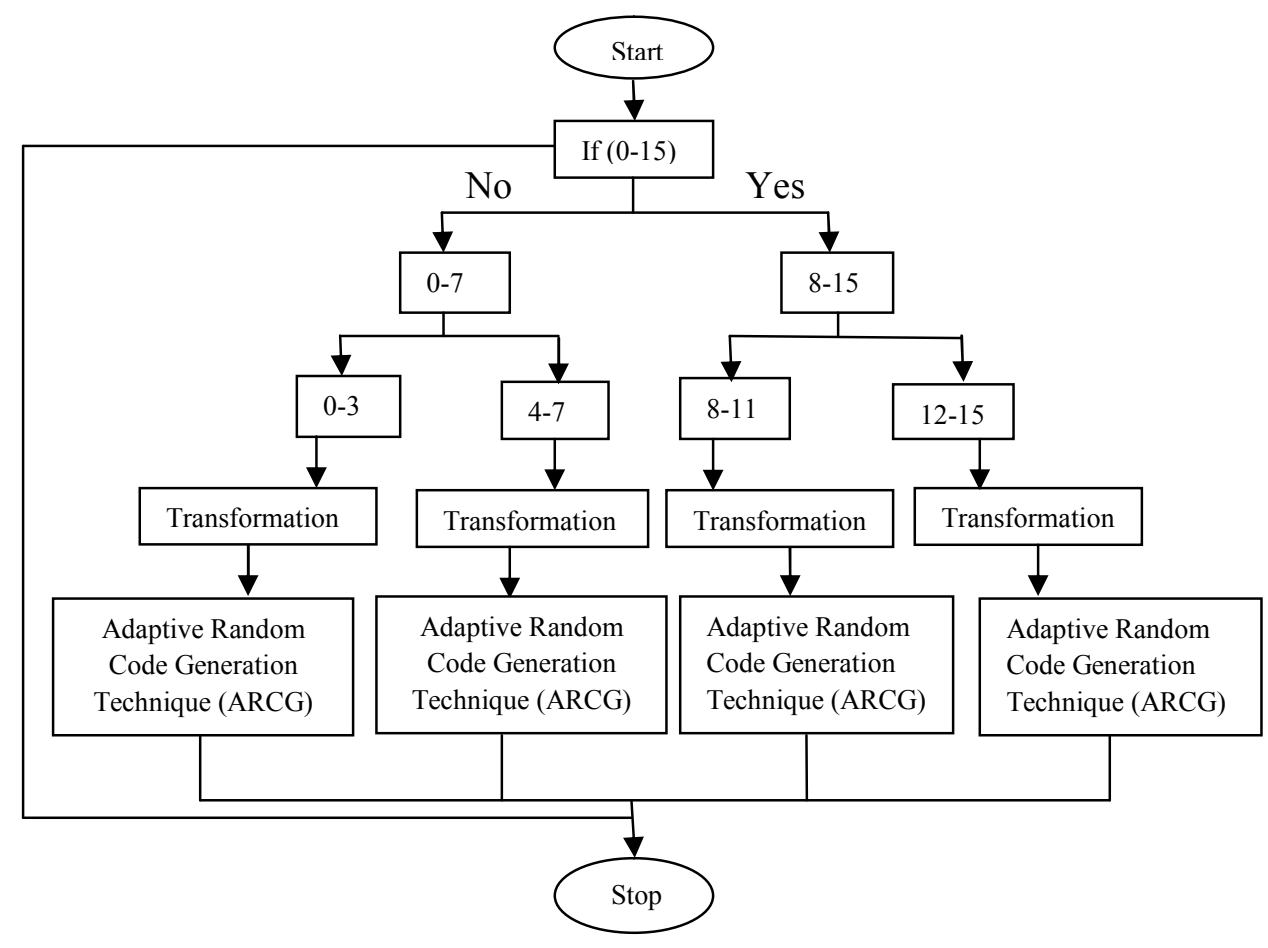

Figure 8: Structural outline of Non Binary Conversion Method

\section{Algorithm explanation:}

Step 1: Start the conversion

Step 2: Enter the input signal. If the signal is $(0-15)$ go to the next step, If it's the signal is not between $(0-15)$ go to the last step.

Step 3: Check the input lies between the bit of 0 to 7 or 8 to 15 .

Step 4: If the input lies between the bit of 0 to 7.

Step 5: Check the input lays between the bit of 0 to 3 or 4 to 7.Transform the input signal and given to the adaptive random code generation technique

Step 6: If suppose the input lies between the bits of 8 to 15 .

Step 7: Check the input lies between 8 to 11 or 12 to 15 .Transform the input signal and given to the adaptive random code generation technique.

Step 8: Stop the conversion. 


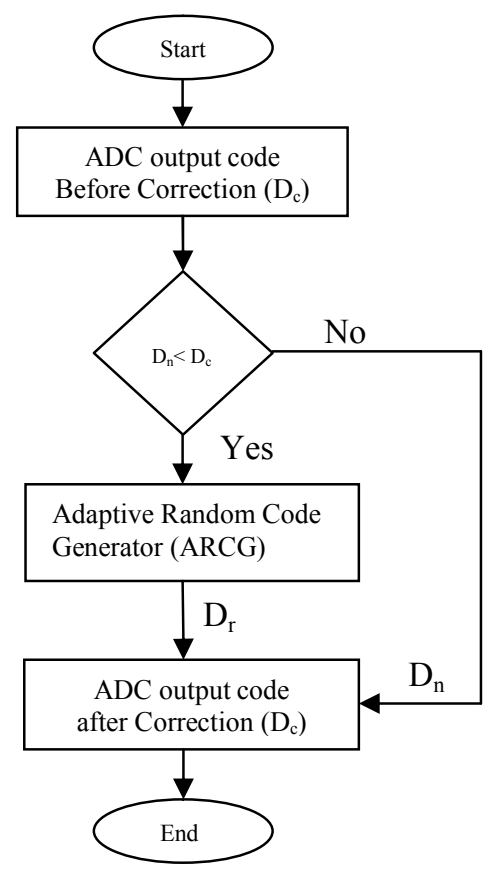

Figure 9: Flow chart for Adaptive Random Code Generator (ARCG)

\subsection{Adaptive Random Code Generation (ARCG) Technique}

The conversion nonlinearity deteriorates when the conventional switching is utilized [14]. Since there is an enormous switching transient in its "down" progress, brought about by switching two capacitors at the same time, the huge switching transient causes the excessive source voltage undershoot just as possibly an overdrive state of the preamplifier, which will at last bring about decision on the comparator's output. Conversely, reversible switching forestalls event of such enormous switching transitory. In each bit cycle, just a single capacitor is changed to acquire the valuable voltage by successive approximation of the source input voltage without wasting energy and settling time. Besides, the mismatches of the depletion capacitor, just as, the steering parasitic capacitance in the internal node of the DAC, cause conversion nonlinearity [15]. 
Ideally, all the quantization levels of the $n$-bit ADC are uniformly spaced, but due to non ideal elements in the actual circuit implementation the code transition points in the transfer function will be moved, which will finally outcome of an abuse decision on the comparator's output [16]. In contrast, reversible switching prevents occurrence of such large switching transitory. To standardize the linearity error, an Adaptive Random Code Generator (ADCG) is proposed, which provides a probable digital post-processing to fix the large quantization errors. This is accomplished by redistributing the steps with statistically equally over the step's \pm LSB range. The flow chart of ADCG technique is depicted in Figure. 9. When the ADCs digital output $D_{\mathrm{n}}$ coordinates any of the digital code in $D_{\mathrm{c}}$, the random code generator will result another digital output $D_{\mathrm{R}}$. The randomized output $D_{\mathrm{R}}$ has one of the three potential qualities $D_{\mathrm{n}}$ and its two contiguous quantization level $D_{n}-1$ and $D_{n}+1$, which are similarly dispersed with an indistinguishable probability of $1 / 3$. The randomized arrangement moves the nonlinearity into expanded average quantization noise power [17]. The digital outputs used to determine the DNL and INL faults are reimbursed values, where the offset of the comparator and linear gain errors will not appear. Along these lines, the immense DNL and INL errors can be calibrated with signal-tonoise distortion ratio (SNDR) of the ADC let fall moderately.

\section{Result Analysis}

\subsection{Simulation Result of QVDC comparator and D-FF}


Table 1 describes about the results analysis of power utilized and transistor required of various comparator techniques. From this table it is noted that the power used for QVDC comparator is reduced by $93.3 \%, 75.7 \%$ and $72.3 \%$ compared to DTC comparator, TS-CMOS-OIC comparator and TS-OLC comparator respectively. The simulation response of QVDC comparator is displayed in Figure. 10. The Comparison Graph of transistor required and power utilized for various comparator techniques $[2,3,5]$ is exposed in Figure. 11. From this graph it is observed that the proposed comparator structure for RSC-DAC based SAR-ADC reduces die area by reducing the transistor requirement by $50 \%, 36 \%$ and $30 \%$ compared to DTC comparator, TS-CMOS-OIC comparator and TS-OLC comparator respectively

Table 1: Result analysis of power utilized and transistor required for various comparator techniques

\begin{tabular}{|c|c|c|}
\hline Comparator Type & Transistor & Power \\
required & Utilized (mW) \\
(DTC) & 14 & 3.32 \\
\hline Two Stage CMOS & & \\
Amplifier with Output & 11 & 2.14 \\
Inverter comparator & & \\
(TS-CMOS-OIC) & & 1.88 \\
\hline Two stage open loop & 10 & \\
Comparator(TS-OLC) & & $\mathbf{0 . 5 2}$ \\
\hline Quantum Voltage & 7 & \\
\hline
\end{tabular}




\begin{tabular}{|c|l|l|}
\hline $\begin{array}{c}\text { Differential Comparator } \\
\text { (QVDC) }\end{array}$ & & \\
\hline
\end{tabular}

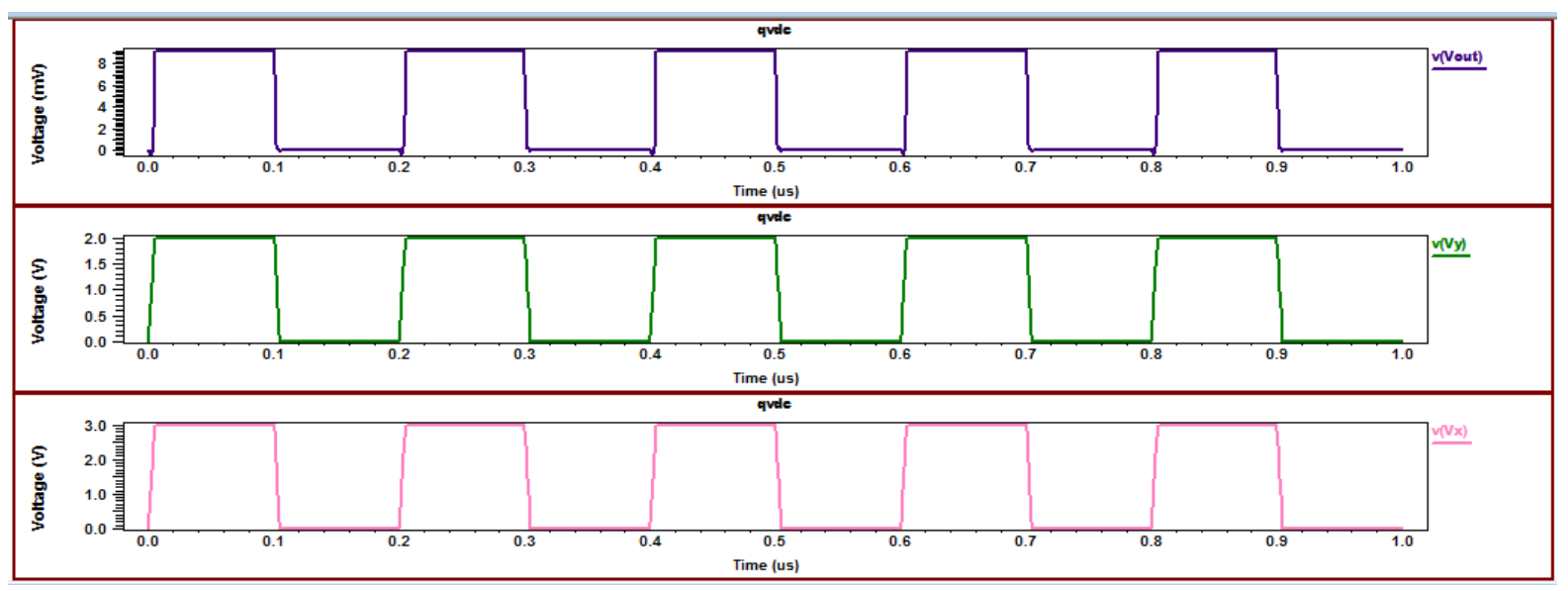

Figure 10: Simulation response of QVDC comparator

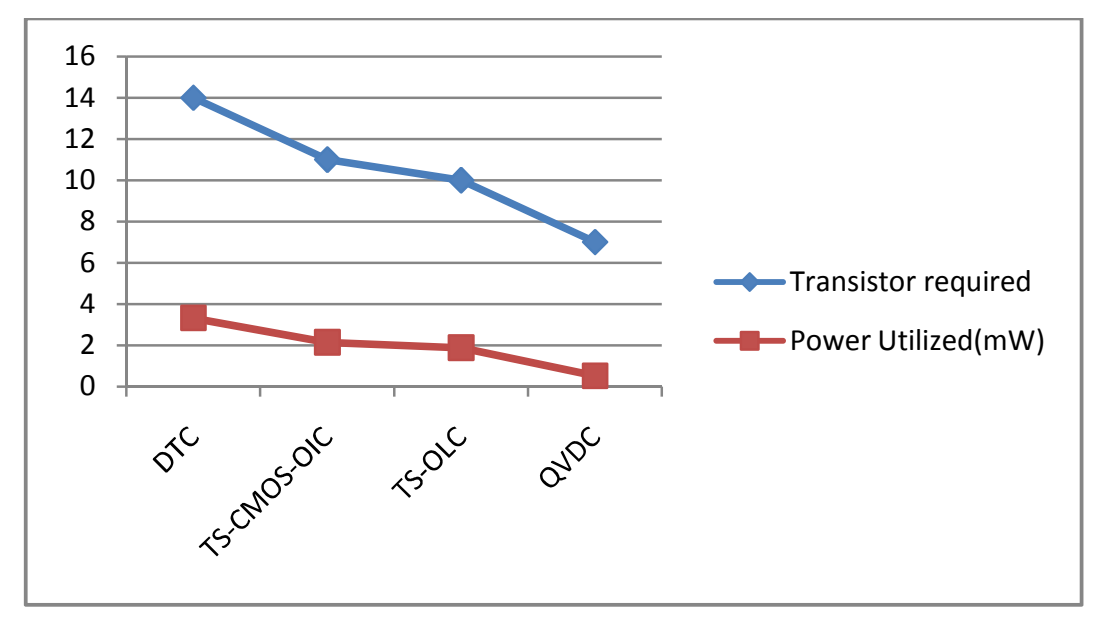

Figure 11: Comparison Graph of transistor required and power utilized for different comparator technique

\subsection{Simulation Result D-FF}


The Simulation result of D-FF is shown in Figure.12. This D-FF used to design the shift register, which control the SAR logic and it consumes $0.21 \mathrm{~mW}$ power and processing delay is 0.19 milliseconds, which is mentioned in Table 3.

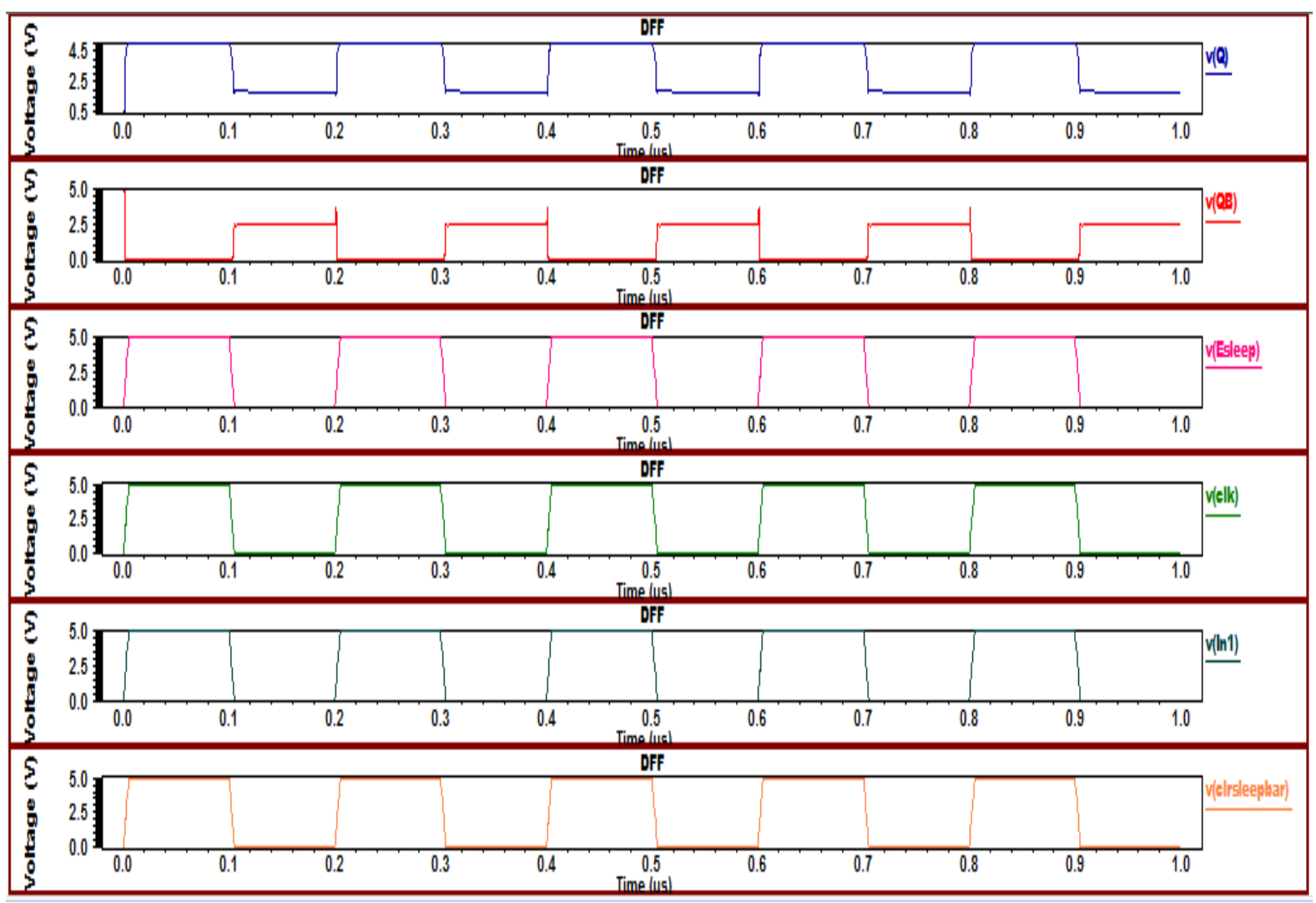

Figure 12: Simulation result of D-FF

5.3 Simulation Result of Reversible Switching Capacitive DAC (RSC-DAC)

For an 8-bit SAR-ADC, the conventional Charge Redistribution Switching Capacitive Digital to Analog Converter (CRSC-DAC) switching technique consumes energy is $339.33 \mathrm{CV}_{\mathrm{r}}^{2}$ (using equation 1) while the suggested RSC-DAC switching method only consumes energy is $21.33 \mathrm{CV}_{\mathrm{r}}^{2}$ (using equation 2). The prospective technique grants about $93 \%$ switching energy, decrease than the regular switching 
scheme. Figure.13 shows the energy reduction comparison chart of various switching schemes. Unexpectedly, the split capacitor switching [8], the energy saving switching [17] and the set-and-down switching [3] methods only provide $37 \%, 56 \%$ and an $81 \%$ reduction respectively.

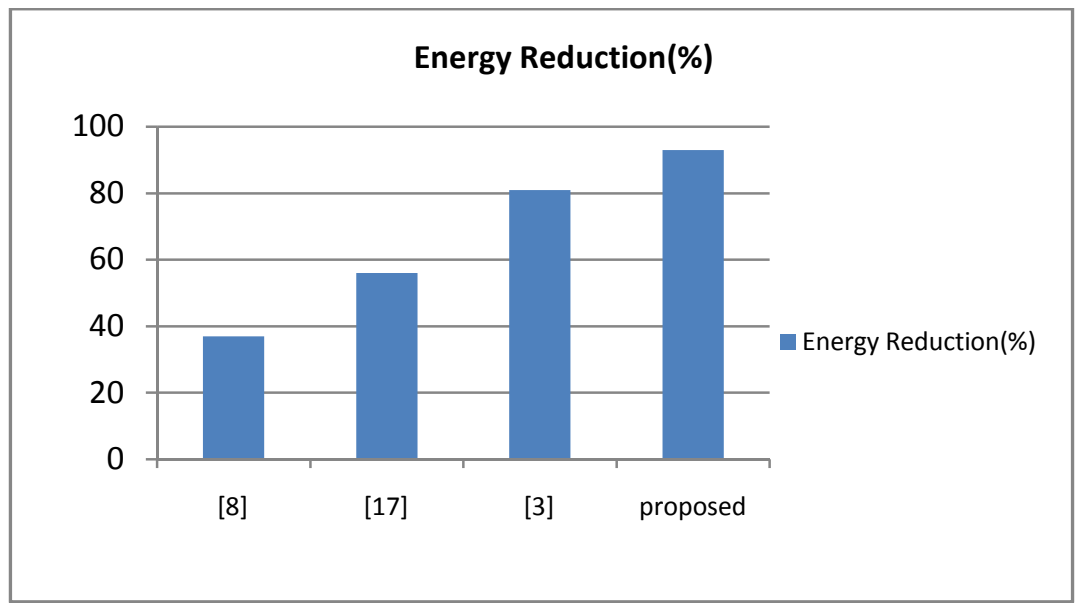

Figure 13: Energy Reduction Comparison chart of various switching schemes

Table 3: Performance analysis of proposed RSC-DAC based SAR-ADC

\begin{tabular}{|l|c|c|}
\hline \multicolumn{1}{|c|}{ Designed structure } & Power utilized & $\begin{array}{c}\text { Propagation } \\
\text { Delay } \mathbf{T}_{\mathbf{p}} \mathbf{( m s )}\end{array}$ \\
\hline QVDC Comparator & 0.13 & 0.11 \\
\hline RSC- DAC & 0.52 & 0.25 \\
\hline D-FF & 0.21 & 0.19 \\
\hline Shift Register based SAR & 0.88 & 0.43 \\
control logic & & $\mathbf{1 . 8 5}$ \\
\hline CRSC-DAC based SAR- & $\mathbf{3 . 0 2}$ & \\
ADC (Conventional) & & \\
\hline
\end{tabular}




\begin{tabular}{|l|c|c|}
\hline RSC-DAC based SAR- & 1.74 & 0.98 \\
ADC(Proposed) & & \\
& & \\
\hline
\end{tabular}

\subsection{Simulation Result of RSC-DAC based SAR-ADC}

The suggested RSC-DAC based SAR-ADC structure reduces the general power usage by $42.4 \%$, and improves the speed by reducing the propagation time delay by $47 \%$, contrasted and the customary CRSC-DAC based SAR-ADC is noted in Table 3. The comparative performance of the constructed SAR-ADC with other techniques is displayed in Table 4. It is shown that the structured RSC-DAC based SAR- ADC could achieve incredible novel execution and a superior compromise among resolution, power consumption, sampling rate, FOM(Figure of Merit), SNDR(Signal to Noise Defined Ratio), SFDR (Spurious Free Dynamic Range), ENOB (Effective Number of Bit)and input frequency in SAR- ADC architectures. The presented work consumes minimum power $1.74 \mathrm{~mW}$ compared with references $[5,6,10,11]$. The Figure Of Merit $\left(\mathrm{FOM}=\right.$ Power/ $\left.\left(2 \times 2^{\mathrm{ENOB}} \mathrm{xRB}\right)\right)$ equals $0.17 \mathrm{pJ} /$ Conversion step, and it has the lowest value compared with the other works $[2,4,6,10,11]$ with an $30 \mathrm{MHz}$ Resolution Bandwidth (RB). The proposed RSC-DAC based SAR-ADC the Differential Nonlinearity (DNL) and Integral Nonlinearity (INL) results are acquired at $10 \mathrm{MHz}$ input frequency. 
Table 4: Comparative Performance of the constructed SAR-ADC with other techniques

\begin{tabular}{|c|c|c|c|c|c|c|c|c|}
\hline Specification & $\begin{array}{l}\text { This } \\
\text { work }\end{array}$ & [2] & [4] & [5] & [6] & [7] & {$[10]$} & [11] \\
\hline $\begin{array}{c}\text { Technology } \\
(\mathrm{nm})\end{array}$ & 250 & 90 & 90 & 65 & 90 & 65 & 180 & 180 \\
\hline $\begin{array}{c}\text { Supply } \\
\text { voltage(volts) }\end{array}$ & 1.5 & 1 & 1 & 1.2 & 0.9 & 0.8 & 1.8 & 1.8 \\
\hline $\begin{array}{c}\text { Resolution(bit } \\
\text { ) }\end{array}$ & 8 & 9 & 8 & 10 & 9 & 10 & 10 & 10 \\
\hline $\begin{array}{c}\text { Power } \\
\text { consumption } \\
(\mathrm{mW})\end{array}$ & 1.74 & 0.82 & $\begin{array}{c}0.06 \\
9\end{array}$ & 4.5 & 13.5 & 0.3 & 9.2 & 21.6 \\
\hline $\begin{array}{c}\text { Sampling } \\
\text { Rate(MS/s) }\end{array}$ & 60 & 40 & $\begin{array}{c}10.2 \\
4\end{array}$ & 100 & 90 & 80 & 50 & 30 \\
\hline DNL(LSB) & $\begin{array}{r}+0.18 / \\
-0.12\end{array}$ & $\begin{array}{c}+0.7 /- \\
0.45\end{array}$ & $\begin{array}{r}+0.3 \\
2 /- \\
0.56\end{array}$ & $\begin{array}{l}+0.1 \\
/-0.1\end{array}$ & $\begin{array}{l}+0.87 / \\
-0.81\end{array}$ & $\begin{array}{l}+0.4 \\
5 / 0.4\end{array}$ & $\begin{array}{l}+0.4 \\
/-0.4\end{array}$ & $\begin{array}{r}+0.5 \\
7 /- \\
0.25\end{array}$ \\
\hline INL(LSB) & $\begin{array}{r}+0.11 / \\
-0.05\end{array}$ & $\begin{array}{c}+0.56 /- \\
0.65\end{array}$ & $\begin{array}{c}0.2 \\
9 /- \\
0.36\end{array}$ & $\begin{array}{l}+0.2 \\
/-0.2\end{array}$ & $\begin{array}{l}+0.71 / \\
-1.55\end{array}$ & $\begin{array}{r}+0.5 \\
8 /- \\
0.74\end{array}$ & $\begin{array}{l}+0.7 \\
/-0.7\end{array}$ & $\begin{array}{l}+0.8 \\
/-0.8\end{array}$ \\
\hline $\begin{array}{l}\text { FOM(pJ/conv } \\
\text { ersion steps })\end{array}$ & 0.17 & 54 & 30 & $\begin{array}{c}0.06 \\
2\end{array}$ & 0.472 & - & 0.29 & 0.26 \\
\hline SNDR(dB) & 46.5 & 51.31 & 48.4 & 59 & 51.8 & 58 & 58 & $\begin{array}{c}56.9 \\
1\end{array}$ \\
\hline
\end{tabular}




\begin{tabular}{|c|c|c|c|c|c|c|c|c|}
\hline SFDR(dB) & $\mathbf{5 1 . 2}$ & - & - & - & 63.4 & - & 74 & 65.9 \\
& & & & & & & & 6 \\
\hline ENOB(bit) & $\mathbf{7 . 4 3}$ & 8.23 & 7.74 & 9.3 & - & $8.3-$ & 9.3 & 9.1 \\
& & & & & & 9.6 & & \\
\hline Fin(MHz) & $\mathbf{1 0}$ & - & 5.00 & 200 & 1.9 & 40 & 6 & 30 \\
& & & 75 & & & & & \\
\hline
\end{tabular}

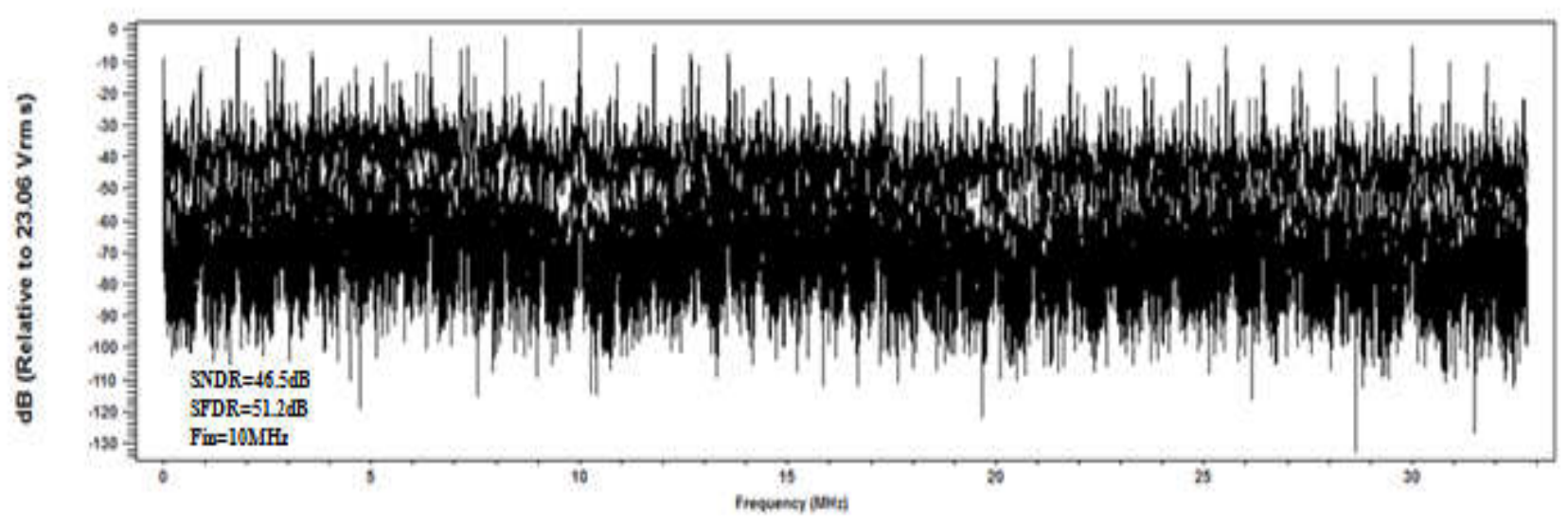

Figure 14: FFT Output spectrums at RB $=30 \mathrm{MHz}$.

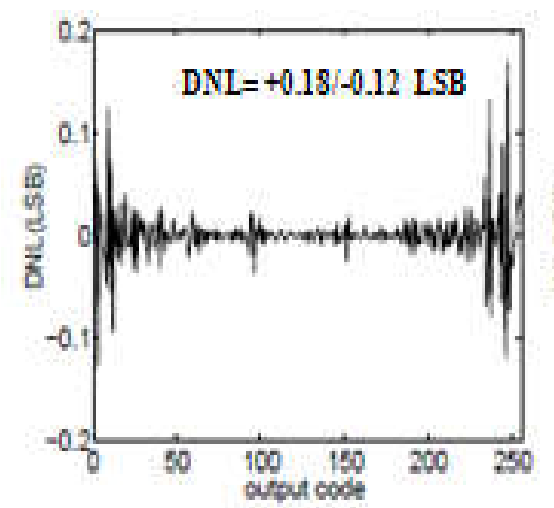

(a)

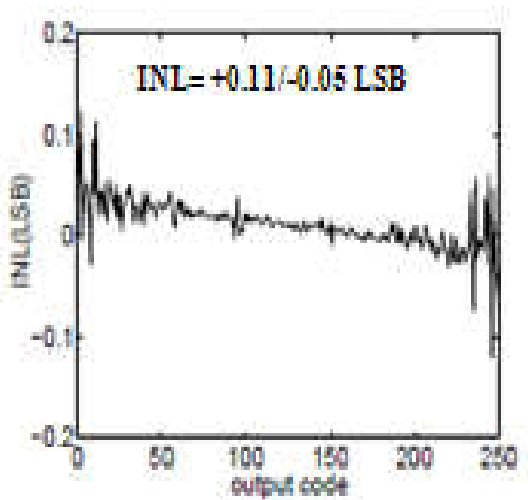

(b)

Figure 15: (a) DNL and (b) INL an input frequency $10 \mathrm{MHZ}$

\section{Conclusion}


A $1.5 \mathrm{~V}$ supply is given to proposed SAR-ADC and has the resolution 8-bit level. The designed RSC-DAC based SAR-ADC system, both delay and switching power of the circuit is reduced due to the efficient reversible switching circuit with ARGC and non binary conversion algorithm. This operation completed in 0.98 milliseconds and consumes $1.74 \mathrm{~mW}$ power. The proposed structure is implemented and Verified by a 250nm CMOS Technology. Using the compact Quantum Voltage Differential Comparator (QVDC), high energy efficient Reversible Switching Capacitive - Digital to Analog Converter (RSC-DAC) and SAR control logic, the constructed design achieves the sampling rate $60 \mathrm{MS} / \mathrm{s}$. The FFT Output spectrum obtained at the $30 \mathrm{MHz}$ resolution bandwidth $(\mathrm{RB})$ is shown in Figure.14. From the above FFT Output spectrum, the deliberate Spurious Free Dynamic Range (SFDR) 51.2dB FS (Full Scale) was noted from the eleventh harmonic and SNDR obtained from FFT was $46.5 \mathrm{~dB}$ with the ENOB (Effective Number of Bit) is 7.43.Figure. 15 summarize the deliberate DNL and INL versus output code and the values of DNL and INL are $+0.18 /-0.12$ LSB and $+0.11 /-0.05$ LSB respectively.

\section{Competing interests}

The authors declare that they have no competing interests.

\section{Author's Contribution}

G. Prathiba and M. Santhi has developed an innovative idea, Analysis Of Reversible Switching Capacitive (RSC) DAC Based Low Power SAR-ADC. G.Prathiba constructs and develops the graphical response for a QVDC comparator, RSC-DAC and SAR control logic circuit. M. Santhi is reviewing the schematic, 
simulation output and Finalizing the manuscript. Authors are studied and approved the completed manuscript.

\section{Acknowledgments}

Not applicable

\section{References}

[1] J. Craninckx and G. V. Plas, “A 65 fJ/conversion-step 0-to-50 MS/s 0-to-0.7 mW 9b charge-sharing SAR ADC in $90 \mathrm{~nm}$ digital CMOS," in IEEE Int. Solid-State Circuits Conf., Dig. Tech. Papers, Feb. 2007, pp. 246-247.

[2] V. Giannini, P. Nuzzo, V. Chironi, A. Baschirotto, G. Van der Plas, and J. Craninckx, "An $820 \mu \mathrm{W}$ 9b $40 \mathrm{MS} / \mathrm{s}$ noise-tolerant dynamic-SAR ADC in $90 \mathrm{~nm}$ digital CMOS," in IEEE Int. Solid-State Circuits Conf., Dig. Tech. Papers, Feb. 2008, pp. $238-610$.

[3] C. C. Liu, S.-J. Chang, G.-Y. Huang, and Y.-Z. Lin, “A $0.92 \mathrm{~mW}$ 10-bit 50-MS/s SAR ADC in $0.13 \mu \mathrm{m}$ CMOS process," in Symp. VLSI Circuits Dig. Tech. Papers, Jun. 2009, pp. 236-237.

[4] P. Harpe, Z. Cui, W. Xiaoyan, G. Dolmans, and H. de Groot, “A 30 fJ/conversionstep 8b 0-to-10 MS/s asynchronous SAR ADC in $90 \mathrm{~nm}$ CMOS," in IEEE Int. SolidState Circuits Conf., Dig. Tech. Papers, Feb. 2010, pp. 388-389.

[5] M. Boulemnakher, E. Andre, J. Roux, and F. Paillardet, “A 1.2V 4.5 mW 10b 100 MS/s pipeline ADC," in IEEE Int. Solid-State Circuits Conf., Dig. Tech. Papers, Feb. 2008, pp. 250-251.

[6] U.-F. Chios, H.-G. Wei, Z. Yan, S. Sai-Weng, U. Seng-Pan, R. P. Martins, and F. Maloberti, "Design and experimental verification of a power effective Flash-SAR sub ranging ADC," in IEEE Trans. Circuits Syst. Aug. 2010. 
[7] Y. Zhu, C.-H. Chan, U.-F. Chio, S.-W. Sin, U. Seng-Pan, and R. P. Martins, “A voltage feedback charge compensation technique for split DAC architecture in SAR ADCs,” in Proc. IEEE ,Circuits Syst., May 2010.

[8] B. P. Ginsburg and A. P. Chandrakasan "An energy-efficient charge recycling approach for a SAR converter with capacitive DAC " in Proc.IEEE, May 2010.

[9] "A self-timing switch-driving register by precharge-evaluate logic for high-speed SAR ADCs," U.-F. Chio, H.-G. Wei, Y. Zhu, S.-W. Sin, U. Seng-Pan, and R. P. Martins, in Proc. IEEE, Dec. 2008.

[10] S. T. Ryu, B. S. Song, and K. Bacrania, “A 10b 50 MS/s opamp-sharing pipeline A/D with current-reuse OTAs," IEEE J. Solid-State Circuits, vol. 42, no. 3, pp. 475485, Mar. 2007.

[11] K. Chandrashekar and B. Bakkaloglu, “A 1.8-V 22-mW 10-bit 30MS/s pipelined CMOS ADC for low-power subsampling applications," IEEE Trans. Very Large Scale Integr. (VLSI) Syst., vol. 19, no. 2, pp. 1610-1616, Sep. 2011.

[12] I. Ahmed, J.Mulder, and D. A. Johns, "A $50 \mathrm{MS} / \mathrm{s} 9.9 \mathrm{~mW}$ pipeline ADC with 58dB SNDR in $0.18 \mu \mathrm{m}$ CMOS using capacitive charge-pumps," in IEEE Int. SolidState Circuits Conf., Dig. Tech. Papers, Feb. 2009, pp. 164-165.

[13] J. Hu, N. Dolev, and B. Murmann, “A 9.4-bit, 50-MS/s, 1.44-mW pipelined ADC using dynamic residue amplification,” in Symp. VLSI Circuits Dig. Tech. Papers, Jun. 2008, pp. 216-217.

[14] O. A. Adeniran, A. Demosthenous, C. Clifton, S. Atungsiri, and R. Soin, "A CMOS low-power ADC for DVB-T and DVB-H systems," in Proc. IEEE Int. Symp. Circuits Syst., vol. 1. May 2004, pp. 209-212. 
[15] Eunji Youn and Young-Chan Jang, "12-bit 20M-S/s SAR ADC using C-R DAC and Capacitor Calibration," in pro. IEEE International SoC Design Conference (ISOCC) Nov. 2018.

[16] Lukang Shi et al, "A 13b-ENOB Noise Shaping SAR ADC with a TwoCapacitor DAC," in in pro. IEEE 61st International Midwest Symposium on Circuits and Systems (MWSCAS), Aug. 2018,pp 153-156.

[17] Y.-K. Chang, C.-S. Wang and C.-K. Wang "A 8-bit 500KS/s low power SAR ADC for bio-medical application,” IEEE Asian Solid-State Circuits Conference, pp. 228-231, Nov. 2008. 
Figures

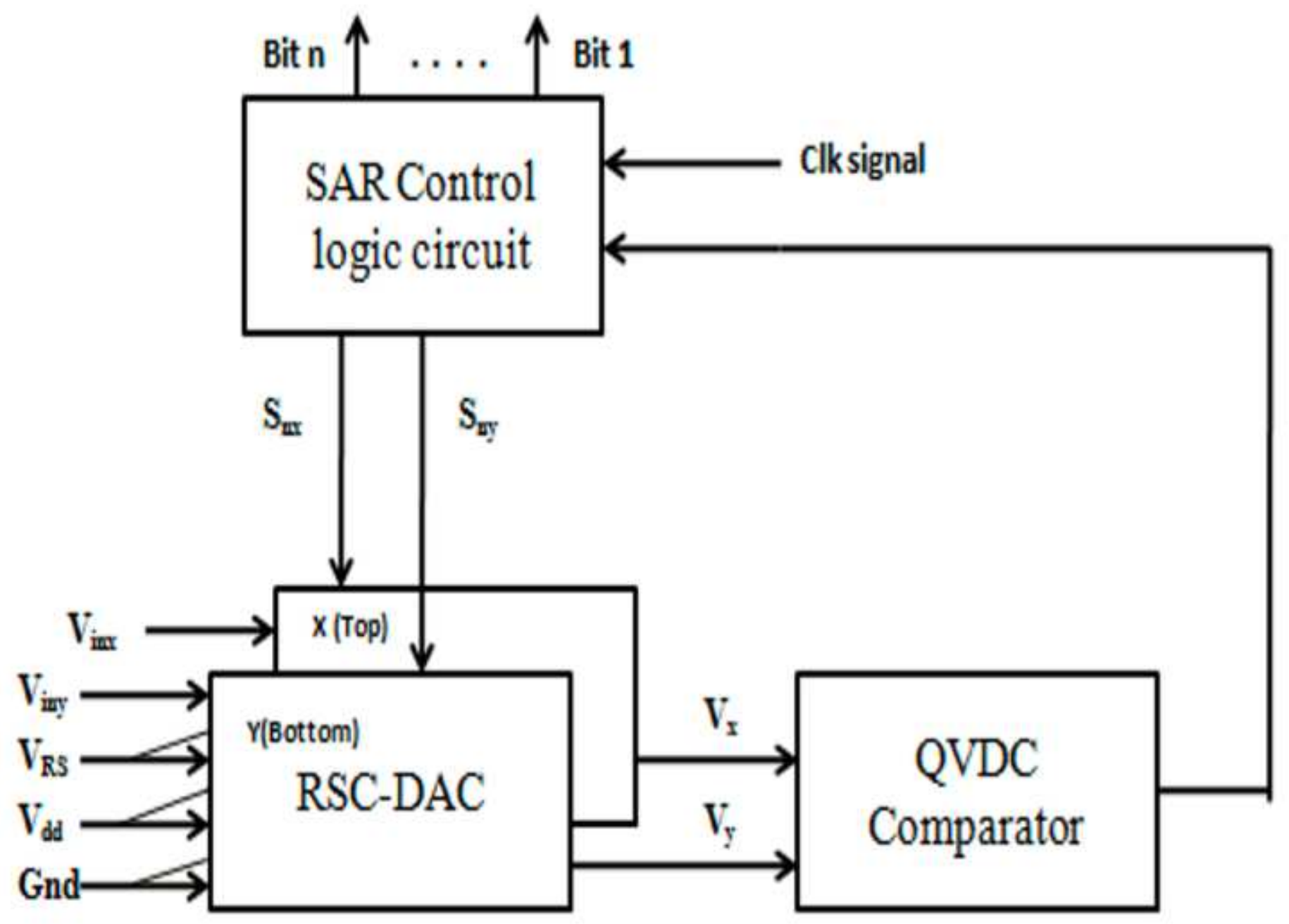

Figure 1

Proposed SAR-ADC Architecture 

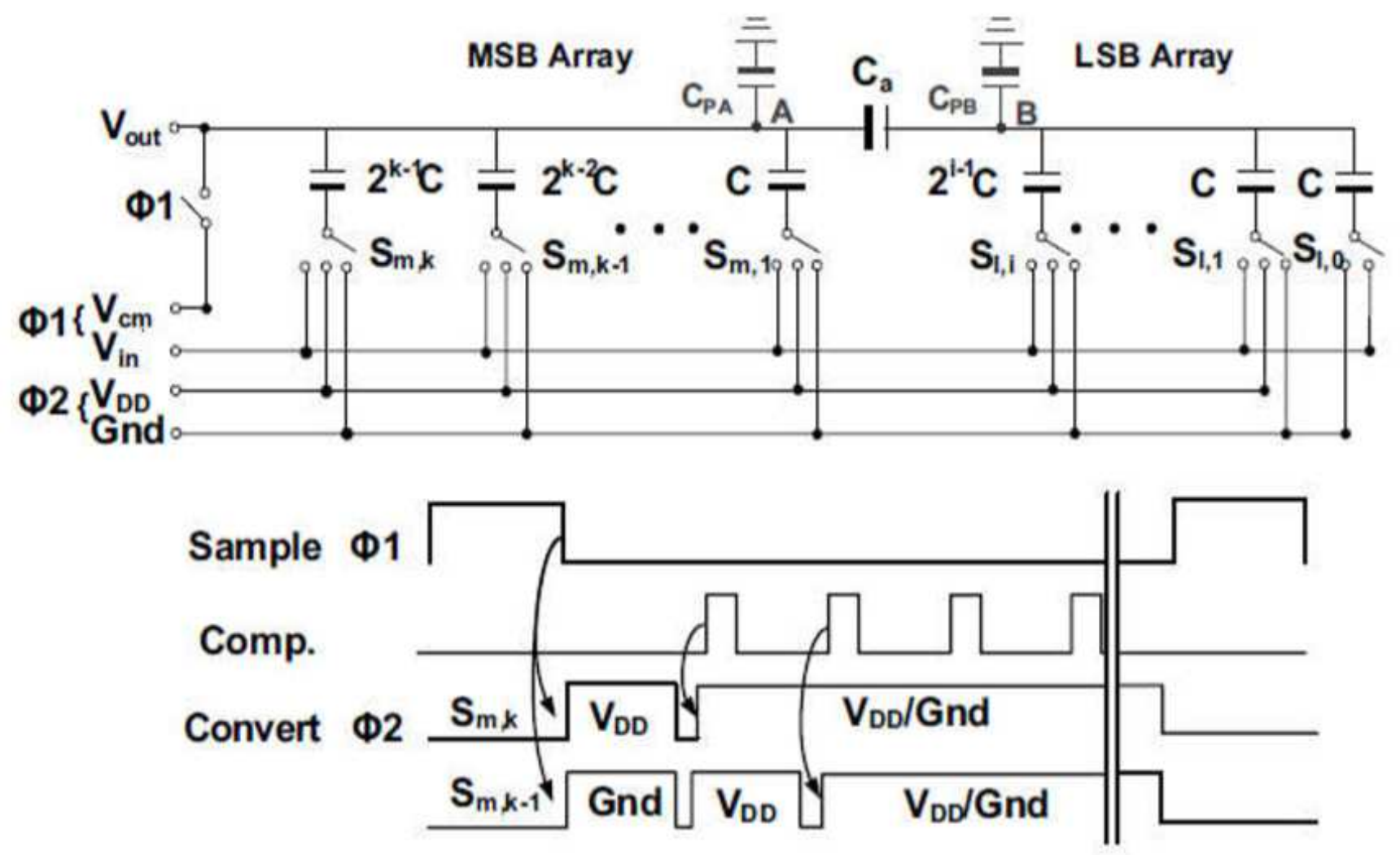

Figure 2

Conventional Charge-Redistribution switching Capacitive DAC 


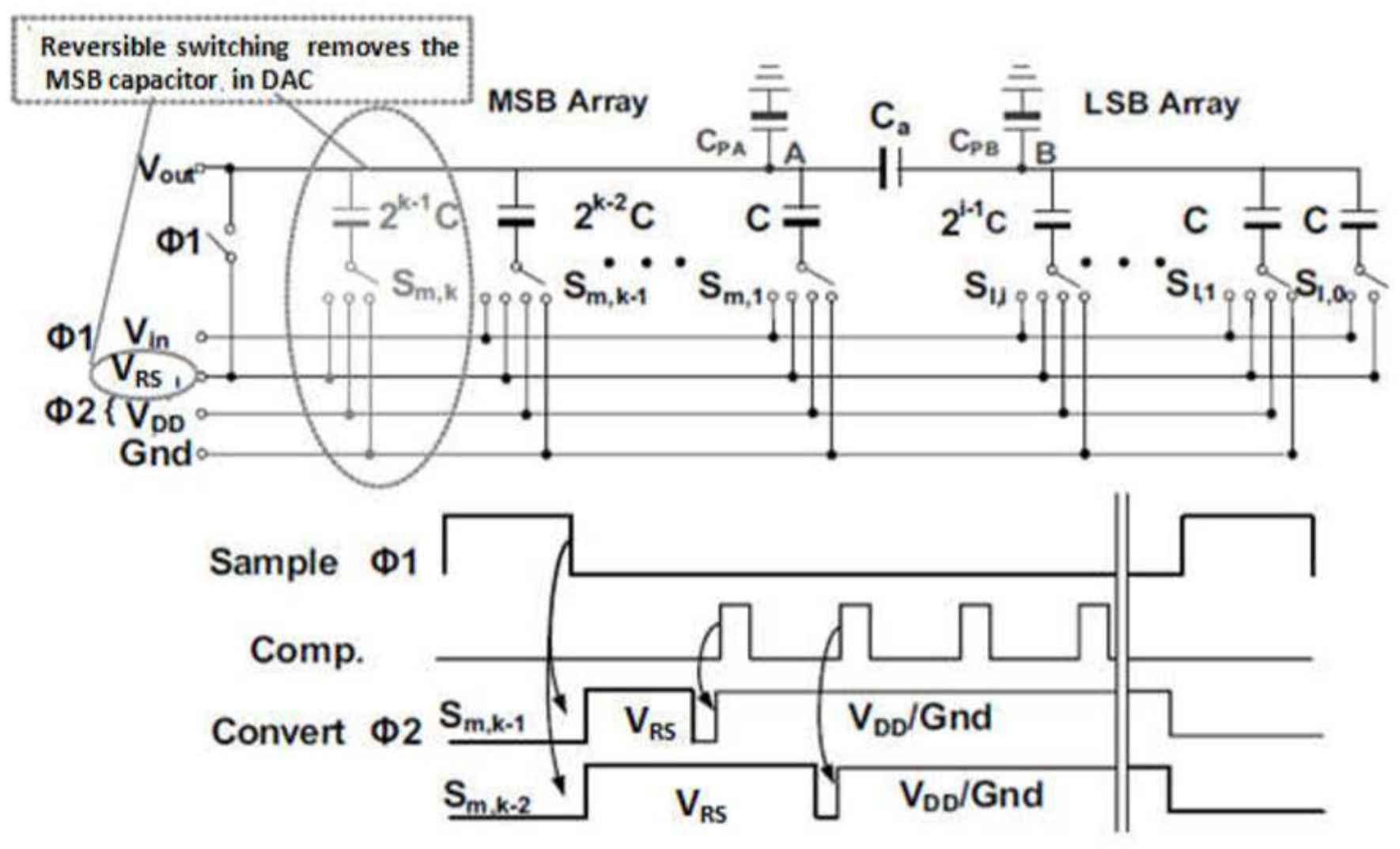

Figure 3

Proposed RSC-DAC 


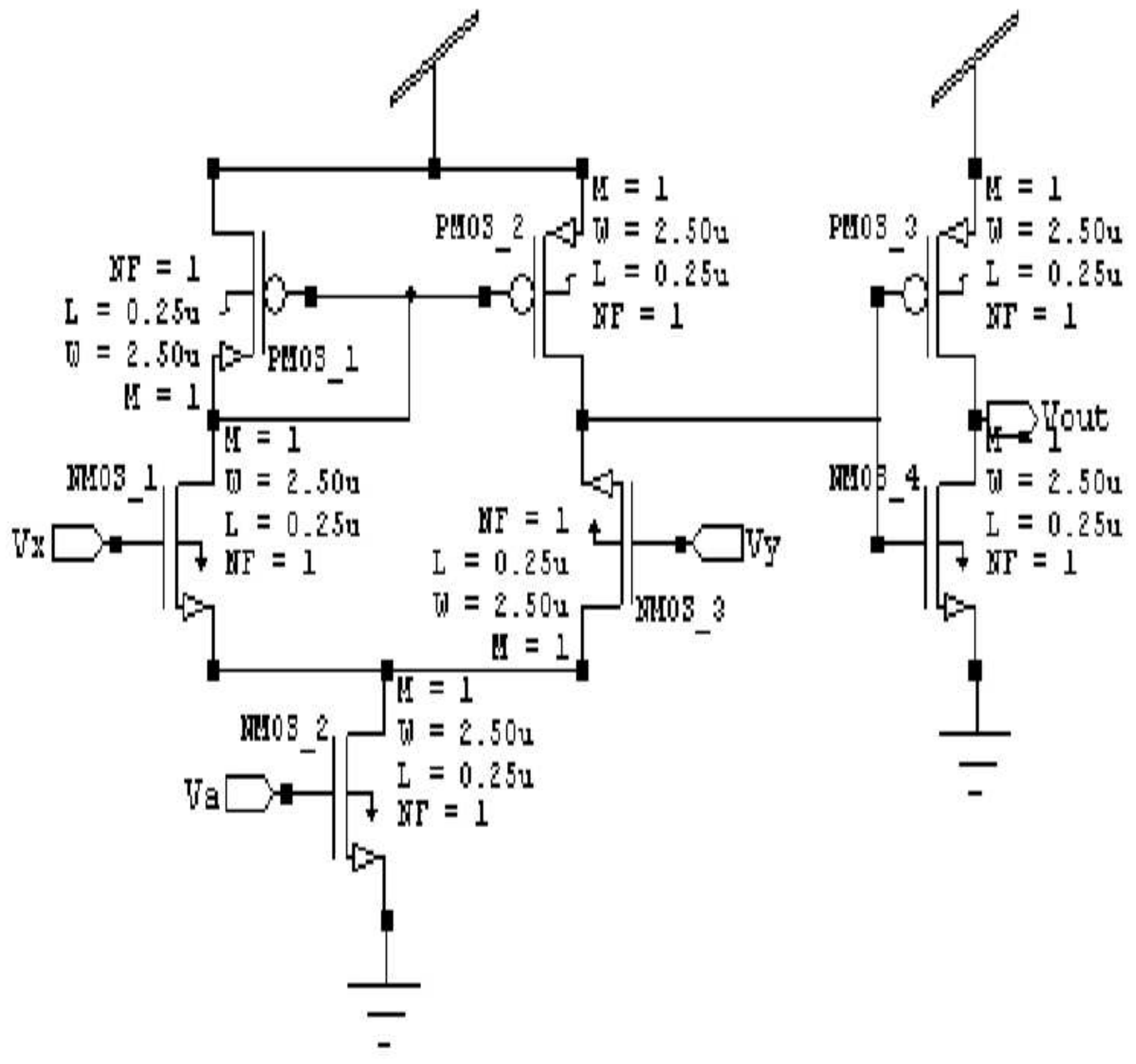

Figure 4

Schematic diagram for QVDC comparator 


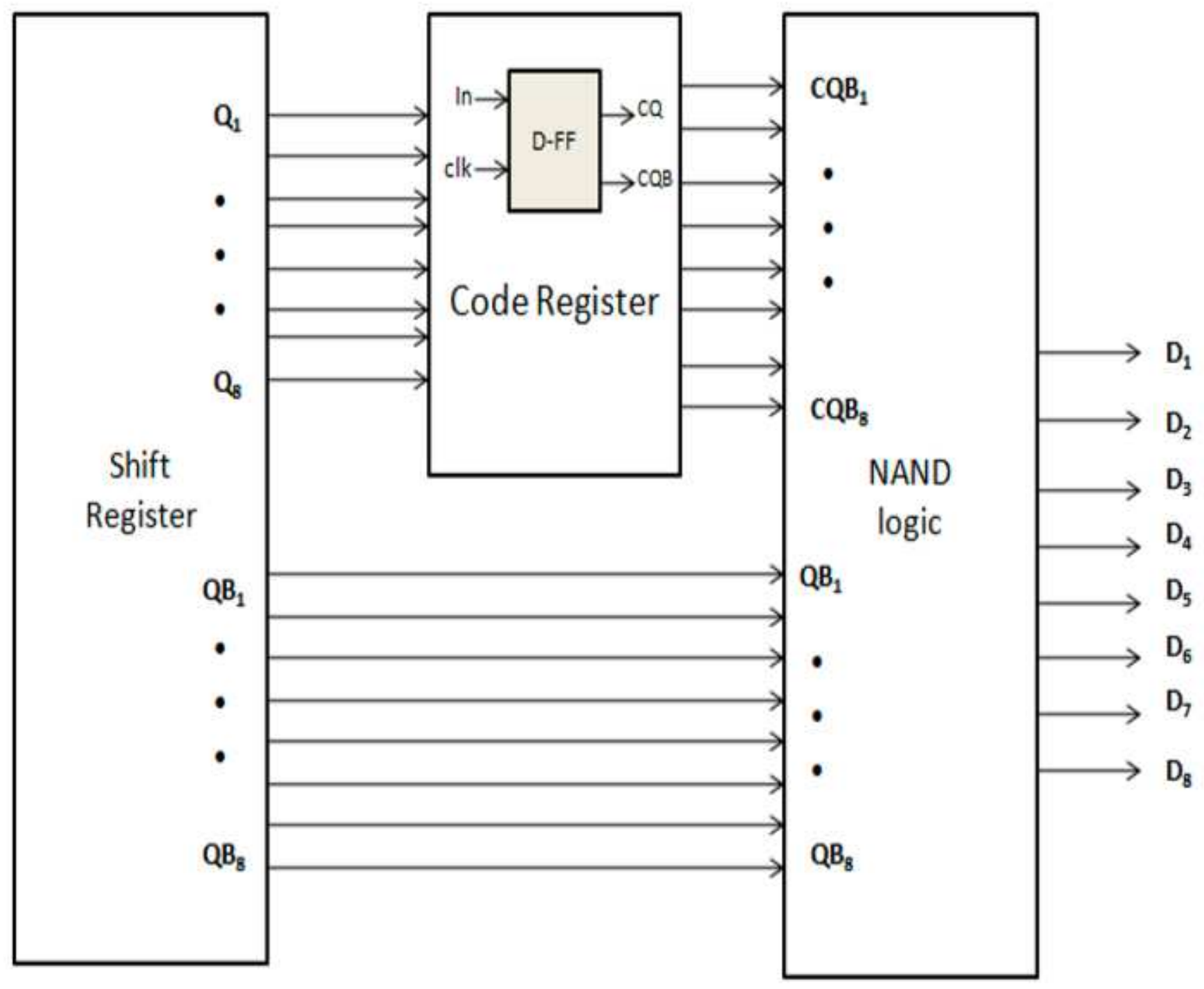

Figure 5

Blocks of SAR control logic 


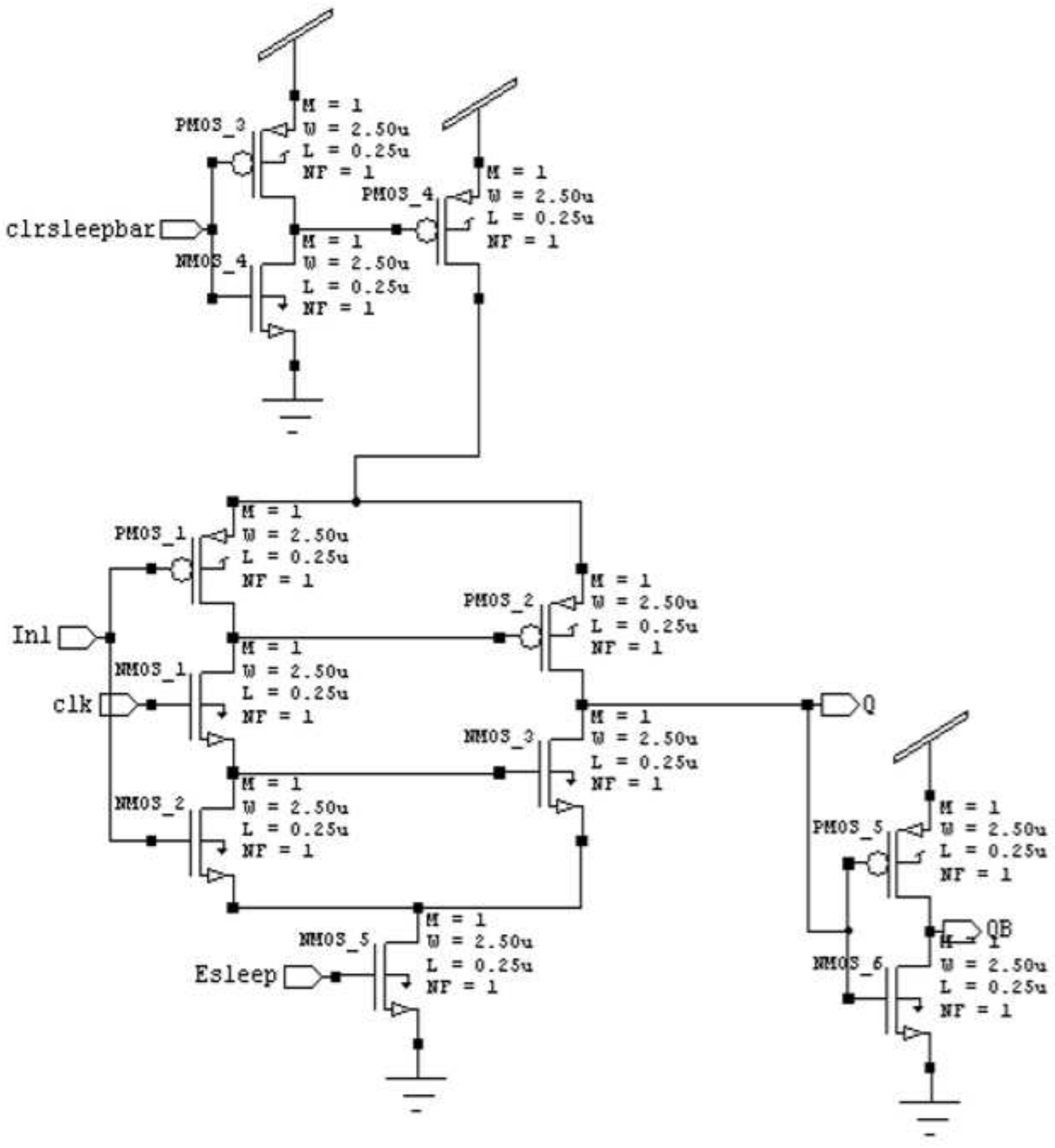

Figure 6

Schematic of D-FF 


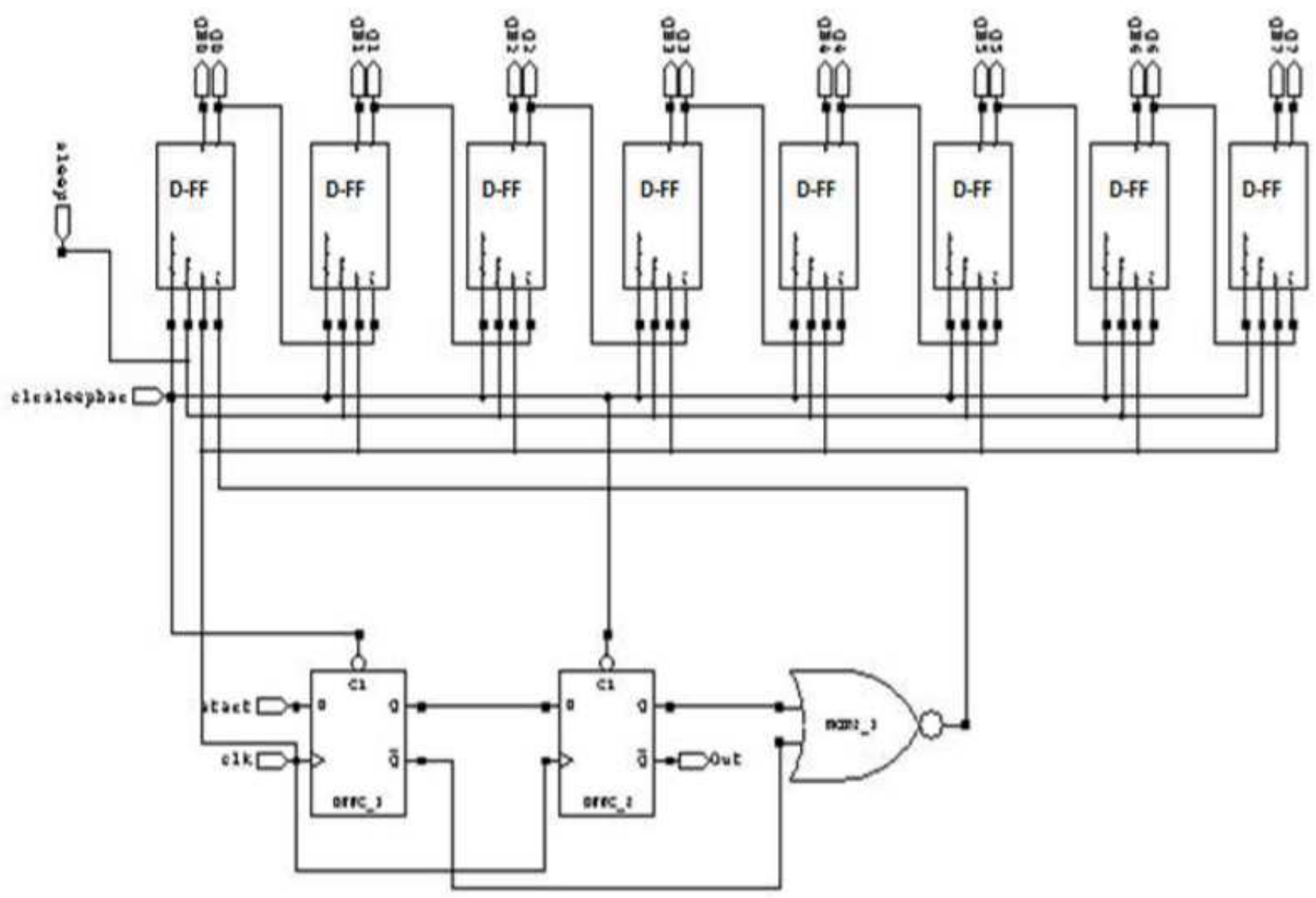

Figure 7

Schematic diagram of Shift register 


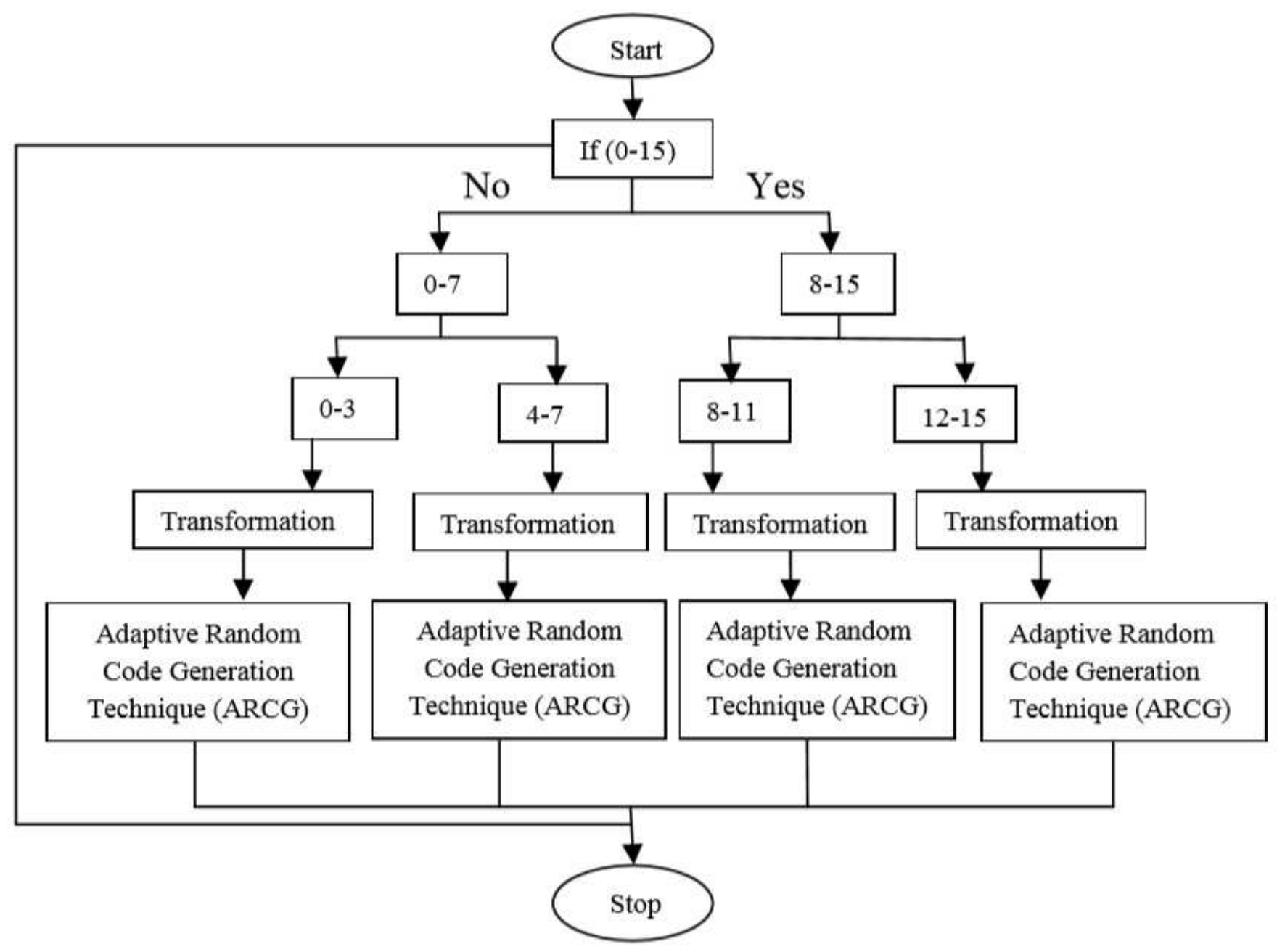

Figure 8

Structural outline of Non Binary Conversion Method 


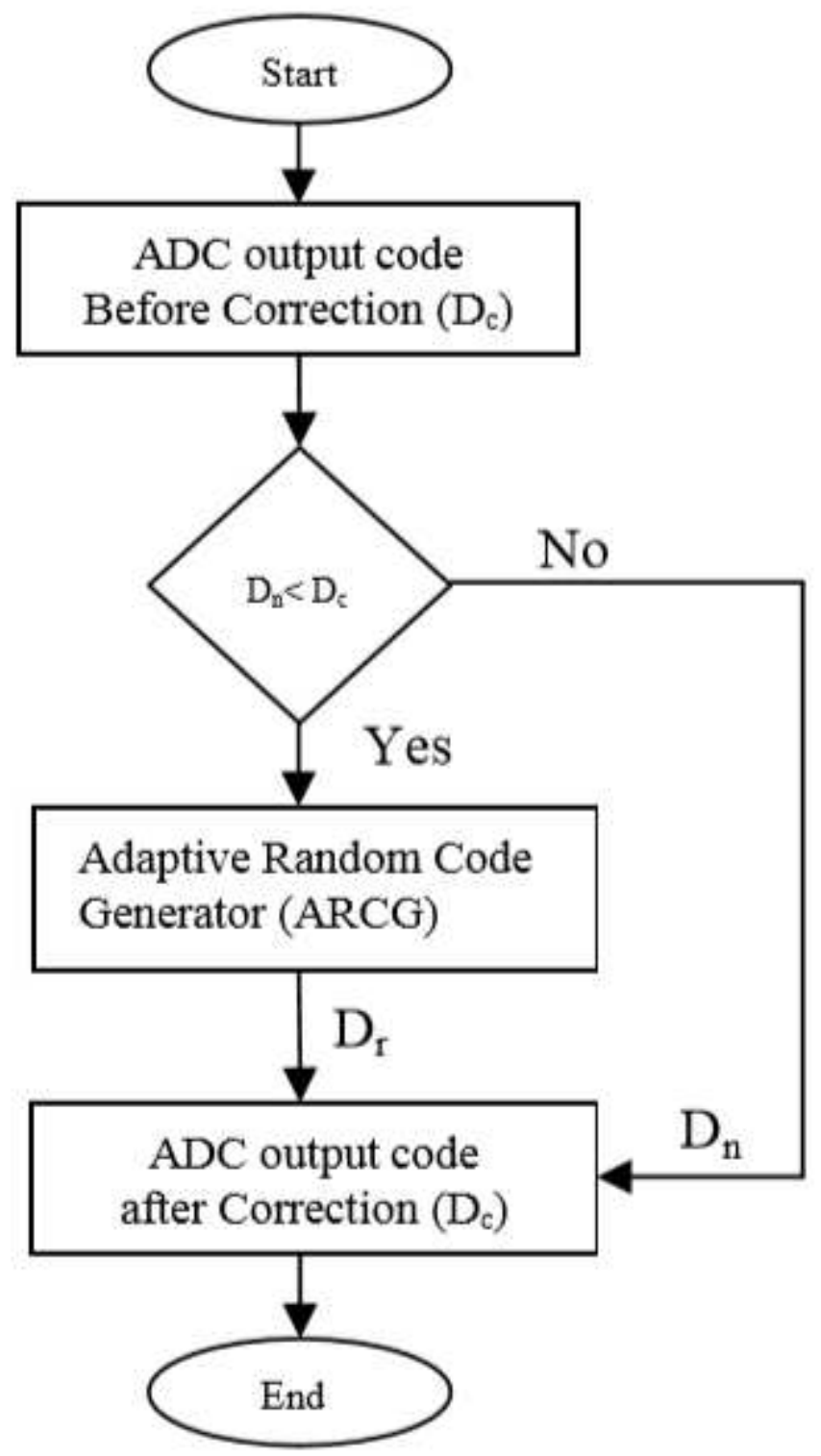

Figure 9

Flow chart for Adaptive Random Code Generator (ARCG) 


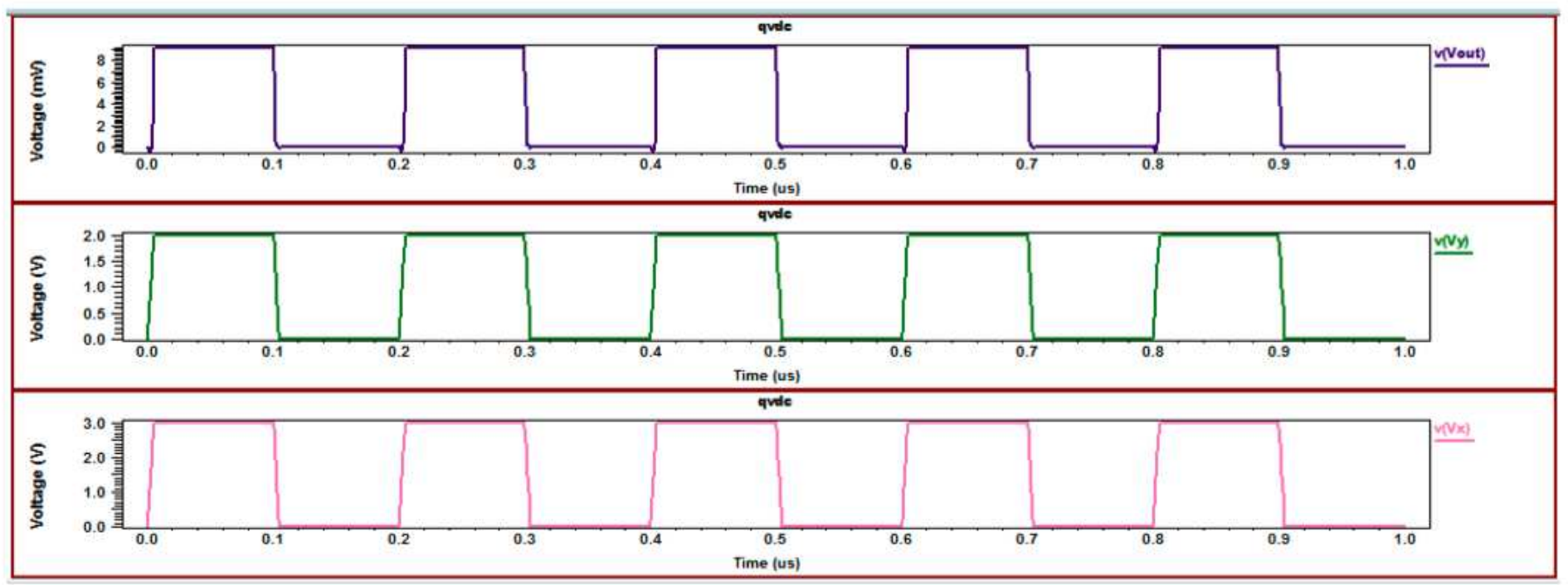

Figure 10

Simulation response of QVDC comparator

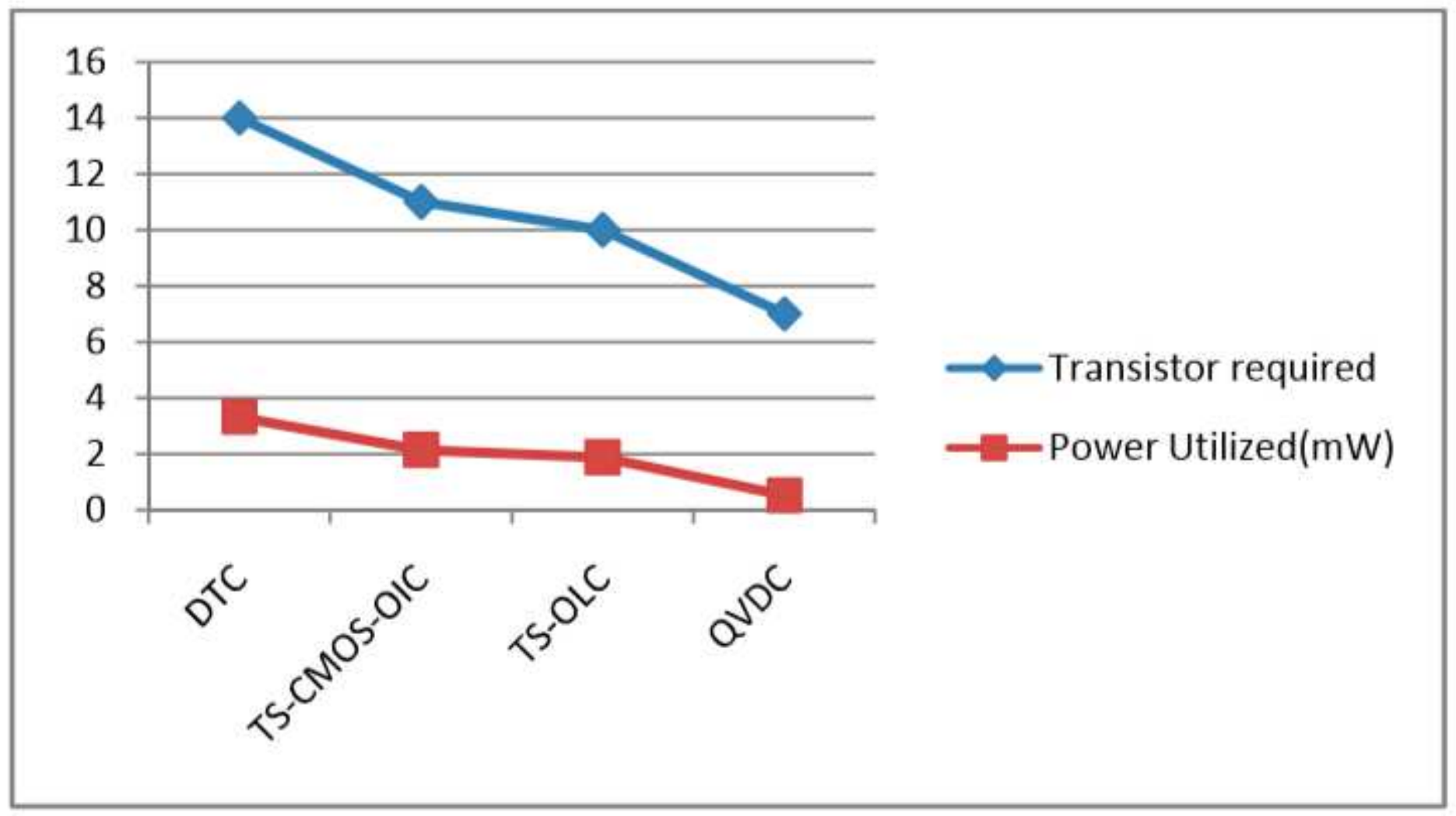

Figure 11

Comparison Graph of transistor required and power utilized for different comparator technique 


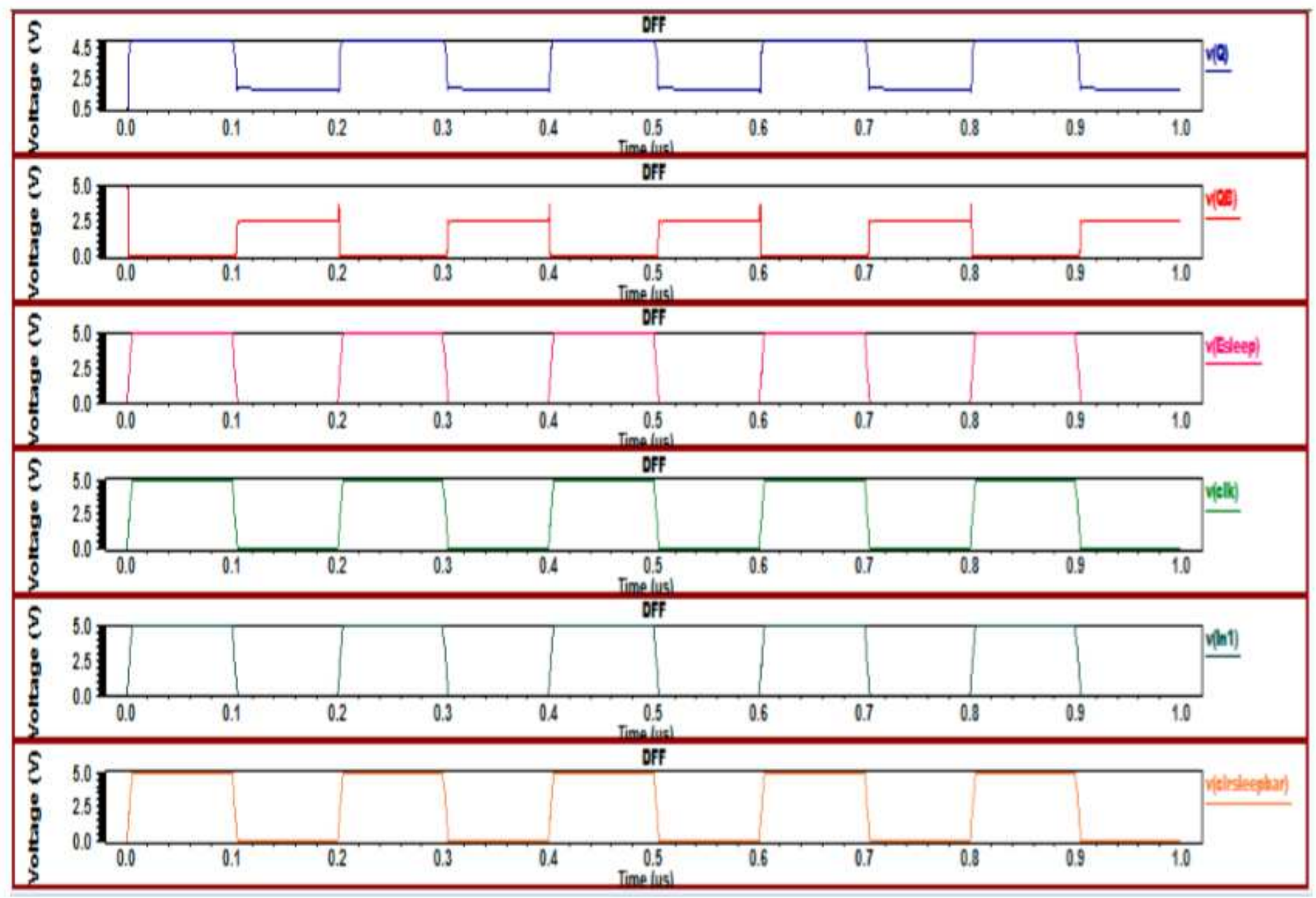

Figure 12

Simulation result of D-FF 


\section{Energy Reduction(\%)}

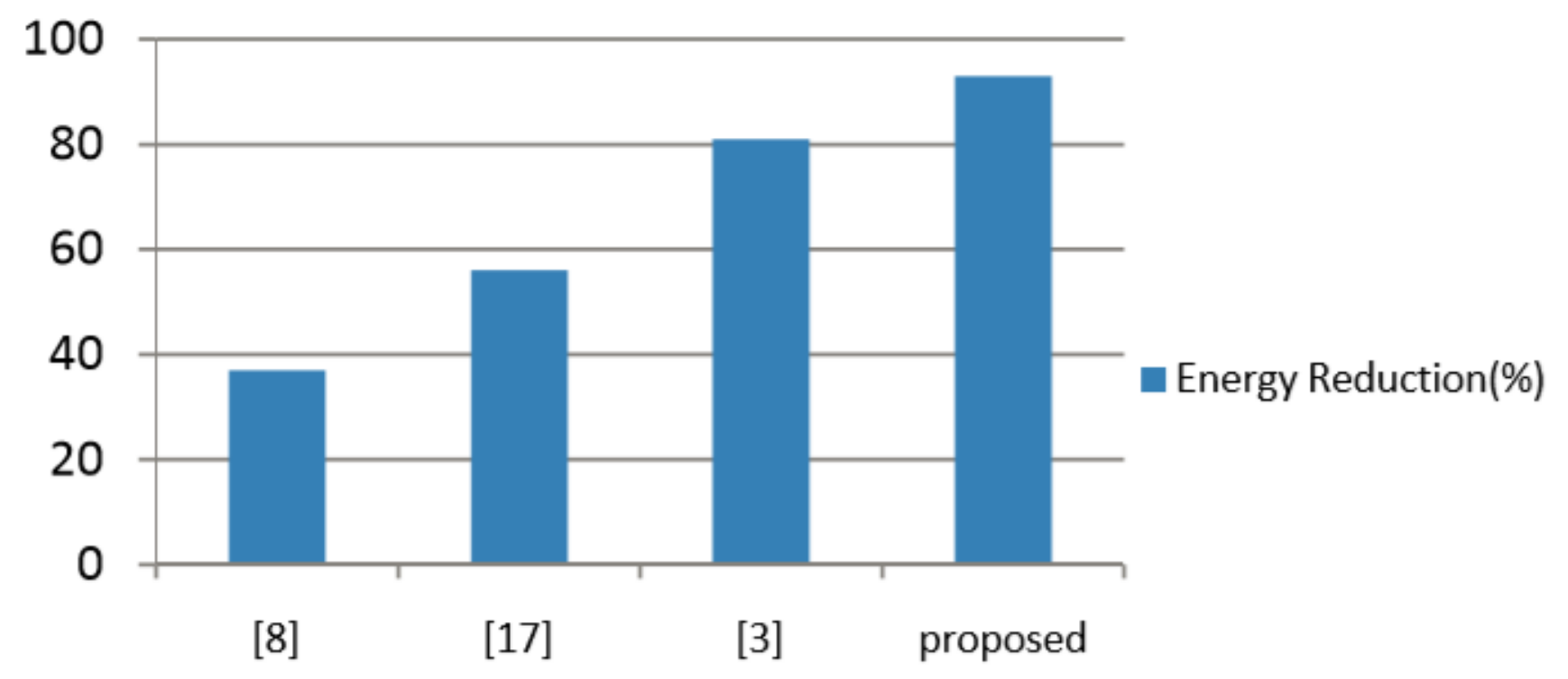

Figure 13

Energy Reduction Comparison chart of various switching schemes

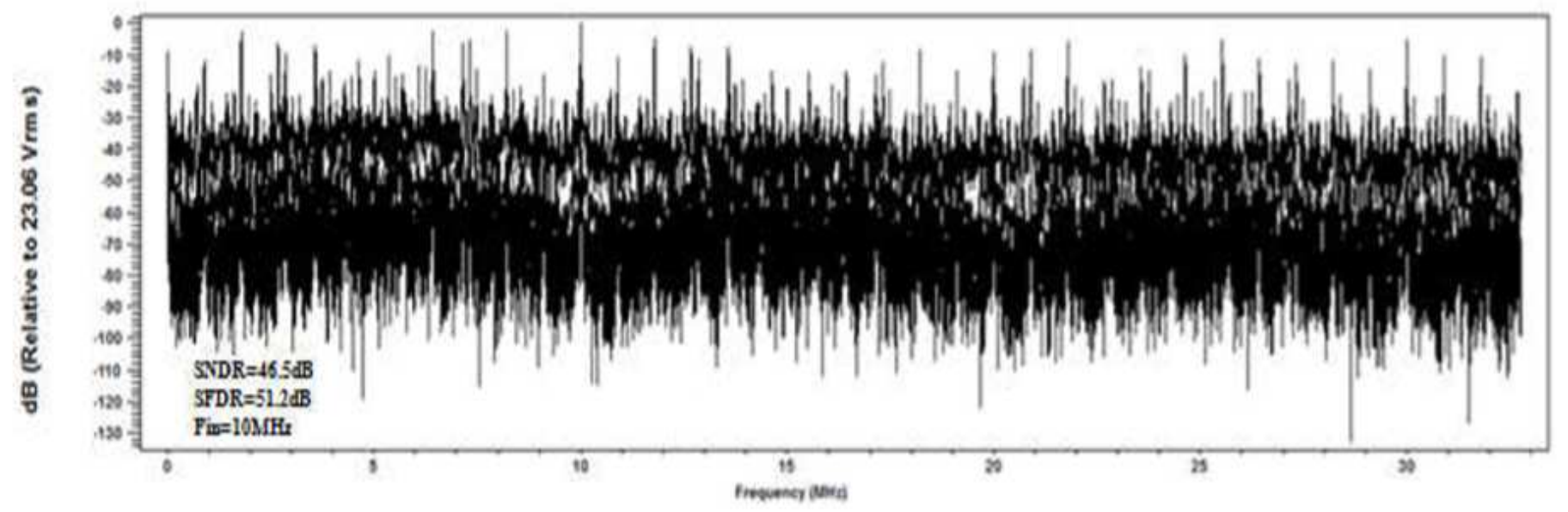

Figure 14

FFT Output spectrums at RB $=30 \mathrm{MHz}$. 


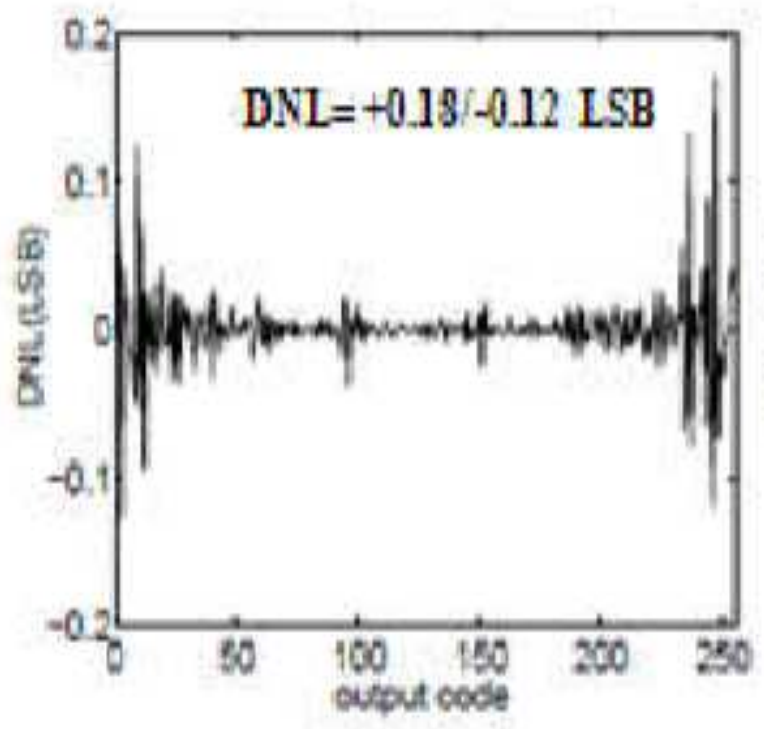

(a)

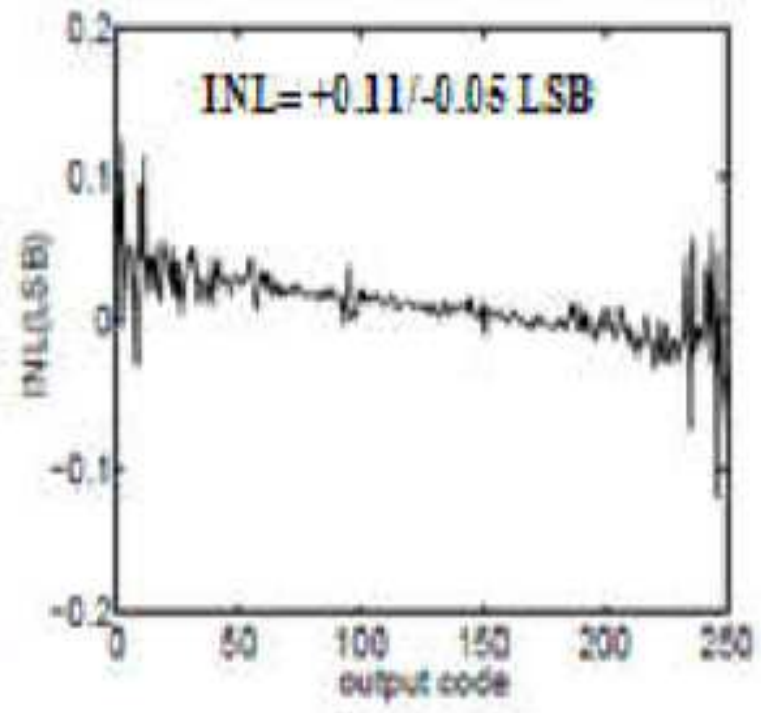

(b)

Figure 15

(a) DNL and (b) INL an input frequency $10 \mathrm{MHZ}$ 